\title{
On the spectral theory of a tapered rod
}

\author{
C. A. Stuart \\ Institut de Mathématiques, EPFL, CH-1015 Lausanne, Switzerland \\ (charles.stuart@epfl.ch)
}

(MS received 9 February 2001; accepted 28 June 2001)

This paper establishes the main features of the spectral theory for the singular two-point boundary-value problem

$$
\begin{gathered}
\left\{A(s) u^{\prime}(s)\right\}^{\prime}+\mu u(s)=0 \quad \text { for all } s \in(0,1], \\
u(1)=\lim _{s \rightarrow 0} A(s) u^{\prime}(s)=0
\end{gathered}
$$

and

$$
\int_{0}^{1} A(s) u^{\prime}(s)^{2} \mathrm{~d} s<\infty
$$

which models the buckling of a rod whose cross-sectional area decays to zero at one end. The degree of tapering is related to the rate at which the coefficient $A$ tends to zero as $s$ approaches 0 . We say that there is tapering of order $p \geqslant 0$ when $A \in C([0,1])$ with $A(s)>0$ for $s \in(0,1]$ and there is a constant $L \in(0, \infty)$ such that $\lim _{s \rightarrow 0} A(s) / s^{p}=L$. A rigorous spectral theory involves relating (1)-(3) to the spectrum of a linear operator in a function space and then investigating the spectrum of that operator. We do this in two different (but, as we show, equivalent) settings, each of which is natural from a certain point of view. The main conclusion is that the spectral properties of the problem for tapering of order $p=2$ are very different from what occurs for $p<2$. For $p=2$, there is a non-trivial essential spectrum and possibly no eigenvalues, whereas for $p<2$, the whole spectrum consists of a sequence of simple eigenvalues. Establishing the details of this spectral theory is an important step in the study of the corresponding nonlinear model. The first function space that we choose is the one best suited to the mechanical interpretation of the problem and the one that is used for treating the nonlinear problem. However, we relate this formulation in a precise way to the usual $L^{2}$ setting that is most common when dealing with boundary-value problems.

\section{Introduction}

In this paper we develop a rigorous spectral theory for the boundary-value problem

$$
\begin{gathered}
\left\{A(s) u^{\prime}(s)\right\}^{\prime}+\mu u(s)=0 \quad \text { for all } s \in(0,1], \\
u(1)=\lim _{s \rightarrow 0} A(s) u^{\prime}(s)=0
\end{gathered}
$$

and

$$
\int_{0}^{1} A(s) u^{\prime}(s)^{2} \mathrm{~d} s<\infty
$$

where $\mu \in \mathbb{R}$ and the coefficient $A \in C([0,1])$ with $A(s)>0$ for $s \in(0,1]$. Thus the problem may be singular at $s=0$ and we monitor this by introducing the following definition. 
Definition 1.1. A profile with tapering of order $p \geqslant 0$ is a function $A \in C([0,1])$ with $A(s)>0$ for $s \in(0,1]$ for which there is a constant $L \in(0, \infty)$ such that $\lim _{s \rightarrow 0} A(s) / s^{p}=L$.

This terminology comes from the significance of $A$ in models for the buckling of rods with variable cross-sections and will be discussed presently. The boundary conditions (1.2), (1.3) also arise naturally in that context. For $p<2$, we find that the problem resembles a regular Sturm-Liouville problem, but at $p=2$ the spectrum is no longer a sequence of isolated eigenvalues. In fact, for $p=2$, there may be no eigenvalues and the essential spectrum is always non-empty. Before describing the results in more detail, let us recall some of the motivation for studying this eigenvalue problem.

\subsection{Tapered rods}

In the simplest interpretation, the variable $s$ measures arc-length along an inextensible rod whose centreline is a plane curve $\{r(s): 0<s<1\}$ given by $r(1)=(0,0)$ and $r^{\prime}(s)=-(\sin u(s), \cos u(s))$. A force $f(0,-1)$, with $f>0$, is applied at the end $r(0)$. The dependent variable $u(s)$ measures the angle between the tangent at $r(s)$ and the direction $(0,-1)$. We assume that the rod obeys the Bernoulli-Euler bending law

$$
M(s)=E A(s) u^{\prime}(s),
$$

with stiffness $E>0$ [1], where $M(s)$ is the bending moment and we use $A(s)$ to denote the moment of inertia of the cross-section at $r(s)$ about its centroid, perpendicular to the plane of bending. Then the mechanical equilibrium of the rod is expressed by the differential equation

$$
\left\{A(s) u^{\prime}(s)\right\}^{\prime}+\mu \sin u(s)=0 \quad \text { for all } s \in(0,1],
$$

where $\mu=f / E$. Equation (1.1) is the linearization of (1.5) about the trivial solution $u \equiv 0$. The boundary conditions (1.2) mean that the lower end $r(1)$ is clamped vertically upwards, whereas the upper end $r(0)$ is free. Since the energy of any configuration is $E J_{\mu}(u)$, where

$$
J_{\mu}(u)=\int_{0}^{1} \frac{1}{2} A(s) u^{\prime}(s)^{2}-\mu\{1-\cos u(s)\} \mathrm{d} s,
$$

condition (1.3) is just the requirement that an equilibrium configuration must have finite energy.

We now show in more detail how the interesting problem of a heavy rod with variable cross-section buckling under its own weight can also be reduced to the above form by a change of variable. In this case, no force is applied at $r(0)$, but we suppose that the rod is made of a homogeneous material of constant density $\rho>0$. We begin by describing a three-dimensional region that will be used to establish a reference configuration for a rod. Let $B$ be an open bounded subset of $\mathbb{R}^{3}$ whose sections

$$
D(z)=\{(x, y):(x, y, z) \in B\}
$$

have the following properties. 
Notation and conventions.

(i) $D(z) \neq \emptyset \Longleftrightarrow 0<z<1$.

(ii) $D(z)$ is simply connected and $(x, y) \in D(z) \Longleftrightarrow(x,-y) \in D(z)$.

(iii) $(0,0)$ is the centroid of $D(z)$, that is, $\iint_{D(z)} x \mathrm{~d} x \mathrm{~d} y=0$.

(iv) There are functions $S$ and $I \in C([0,1])$ such that, for $0<z<1, S(z)$ is the area of $D(z)$ and $I(z)$ is its moment of inertial about the $y$-axis. Thus $S(z)=\iint_{D(z)} \mathrm{d} x \mathrm{~d} y>0$ and $I(z)=\iint_{D(z)} x^{2} \mathrm{~d} x \mathrm{~d} y>0$ for all $z \in(0,1)$ and we suppose, in addition, that $S(0)>0$ and $I(0)>0$.

We think of $B$ as being occupied by a rod-like body in its reference configuration. A planar configuration of the rod is identified with a curve in the $(x, z)$-plane, which will be taken to be formed by the centroids of these sections. Suppose that the inextensible rod has unit length and consider a smooth planar configuration. We use arc-length, $s$, measured from the free end, $r(0)$, for a parametric representation, $r:[0,1] \rightarrow \mathbb{R}^{2}$, of this configuration. Then there is a unique angle, $\theta(s) \in[0,2 \pi)$, such that

$$
r^{\prime}(s)=-(\sin \theta(s), \cos \theta(s)) .
$$

Choosing axes such that gravity acts in the direction of $(0,-1), \theta(s)$ measures the angle between the tangent to the rod at position $r(s)$ and the gravitational force. Choosing the origin of the coordinates so that $r(1)=(0,0)$, the reference configuration is given by $\theta(s) \equiv 0$ or $r(s)=(0,1-s)$.

Using $M(s)$ to denote the bending moment at $r(s)$, the equilibrium conditions are expressed by the differential equation

$$
M^{\prime}(s)+\rho g\left[\int_{0}^{s} S(1-\tau) \mathrm{d} \tau\right] \sin \theta(s)=0 \quad \text { for } 0<s<1,
$$

where $g>0$ is the gravitational constant and $S(z)$ is the area of the horizontal section $D(z)$ at height $z$ in the reference configuration. Since the end $r(0)$ is free, whereas the other end $r(1)$ is clamped vertically upwards, we must impose the boundary conditions

$$
\lim _{s \rightarrow 0} M(s)=0 \quad \text { and } \quad \theta(1)=0 .
$$

Finally, the Bernoulli-Euler constitutive relation (1.4) for the elastica is expressed as $M(s)=E I(1-s) \theta^{\prime}(s)$, where $E>0$ is a material constant and $I(z)$ is the moment of inertia of the horizontal section at height $z$ in the reference configuration about the axis through $(0,0, z)$ and perpendicular to the $(x, z)$-plane (see eqn $(16.12)$ of $[1])$. The equilibrium equation now becomes

$$
\left\{I(1-s) \theta^{\prime}(s)\right\}^{\prime}+\xi\left[\int_{0}^{s} S(1-\tau) \mathrm{d} \tau\right] \sin \theta(s)=0 \quad \text { for } 0<s<1,
$$

where $\xi=\rho g / E>0$ and $I, S \in C([0,1])$ are given functions. We seek solutions $\theta$ satisfying the boundary conditions (1.6), which become

$$
\lim _{s \rightarrow 0} I(1-s) \theta^{\prime}(s)=0 \text { and } \quad \theta(1)=0 .
$$


Motivated by the work on the shape of the tallest column [4,11], we are particularly interested in cases where $S(1-s)$ and $I(1-s) \rightarrow 0$ as $s \rightarrow 0$, so the first boundary condition in (1.8) cannot be replaced by $\lim _{s \rightarrow 0} \theta^{\prime}(s)=0$. For the same reason, configurations that satisfy the boundary conditions (1.8) do not necessarily have finite elastic energy, so this has to be ensured separately. The total energy of a configuration is given by

$$
\int_{0}^{1} \frac{1}{2} E I(1-s) \theta^{\prime}(s)^{2}-\rho g\left[\int_{0}^{s} S(1-\tau) \mathrm{d} \tau\right]\{1-\cos \theta(s)\} \mathrm{d} s .
$$

Since $S \in C([0,1])$, this energy is finite if and only if

$$
\int_{0}^{1} I(1-s) \theta^{\prime}(s)^{2} \mathrm{~d} s<\infty .
$$

We can now give a precise statement of the mathematical problem to be discussed. Given a constant $\xi>0$ and functions $I$ and $S \in C([0,1])$ with $I(z)$ and $S(z)>0$ for $z<1$, we seek solutions of (1.7) that satisfy (1.8) and (1.9). The following change of variables brings this problem into a more convenient form, which coincides with the problem of a loaded rod discussed earlier. Given a function $S \in C([0,1])$ with $S(z)>0$ for $z<1$, let

$$
Z=\int_{0}^{1} \int_{0}^{\sigma} S(1-\tau) \mathrm{d} \tau \mathrm{d} \sigma \quad \text { and } \quad t=t(s)=\frac{1}{Z} \int_{0}^{s} \int_{0}^{\sigma} S(1-\tau) \mathrm{d} \tau \mathrm{d} \sigma .
$$

Then set

$$
u(t)=\theta(s) \quad \text { and } \quad A(t)=I(1-s) \int_{0}^{s} S(1-\tau) \mathrm{d} \tau \quad \text { where } t=t(s) .
$$

Clearly, $t$ increases from 0 to 1 as $s$ increases from 0 to 1 . Furthermore,

$$
\begin{aligned}
\int_{0}^{1} I(1-s) \theta^{\prime}(s)^{2} \mathrm{~d} s & =\frac{1}{Z} \int_{0}^{1} A(t) u^{\prime}(t)^{2} \mathrm{~d} t \\
A(t) u^{\prime}(t) & =Z I(1-s) \theta^{\prime}(s) \quad \text { where } t=t(s),
\end{aligned}
$$

equation (1.7) becomes

$$
\left\{A(t) u^{\prime}(t)\right\}^{\prime}+\mu \sin u(t)=0 \quad \text { for } 0<t<1,
$$

where $\mu=Z^{2} \xi$, and the energy becomes

$$
\frac{E}{Z} \int_{0}^{1} \frac{1}{2} A(t) u^{\prime}(t)^{2}-\mu\{1-\cos u(t)\} \mathrm{d} t .
$$

Equation (1.1) is the linearization of (1.14) and conditions (1.8) and (1.9) reduce to $(1.2)$ and $(1.3)$, but the independent variable is no longer the arc-length along the curve of centroids of the rod.

To interpret our results, we should relate tapering of order $p$ for the coefficient $A$ to the physical variables $I$ and $S$. Note that if

$$
\lim _{s \rightarrow 0} \frac{S(1-s)}{s^{q}}=K>0 \text { and } \lim _{s \rightarrow 0} \frac{I(1-s)}{s^{r}}=J>0,
$$


then

$$
\lim _{t \rightarrow 0} \frac{A(t)}{t^{p}}=L \quad \text { where } p=\frac{r+q+1}{q+2} \text { and } L=\frac{J K}{q+1}\left\{\frac{Z(q+1)(q+2)}{K}\right\}^{p} .
$$

In particular, in the case of a uniform column where $S$ and $I$ are constant, $q=r=0$ and hence $p=\frac{1}{2}$.

In the discussion of tapered columns buckling under their own weight $[4,8,11]$, it is often assumed that the sections are all similar, since, in this case, $I$ is proportional to $S^{2}$. More precisely, in addition to the assumptions (i)-(iv), we suppose that the sections $D(z)$ have the following property.

(v) There is a function $\alpha \in C([0,1])$ with $\alpha(z)>0$ for $z<1$ and a set $D \subset \mathbb{R}^{2}$ such that $D(z)=\alpha(z) D$ for all $z \in(0,1)$.

Then

$$
S(z)=\alpha(z)^{2}|D|, \quad \text { where }|D| \text { is the area of } D
$$

and

$$
I(z)=C S(z)^{2}, \quad \text { where } C=\frac{1}{|D|^{2}} \iint_{D} x^{2} \mathrm{~d} x \mathrm{~d} y .
$$

Under these conditions, $r=2 q$ in $(1.16)$,

$$
A(t)=C S(1-s)^{2} \int_{0}^{s} S(1-\tau) \mathrm{d} \tau
$$

and, if

$$
\lim _{s \rightarrow 0} \frac{S(1-s)}{s^{q}}=K>0
$$

then

$$
\lim _{t \rightarrow 0} \frac{A(t)}{t^{p}}=L, \quad \text { where } p=\frac{3 q+1}{q+2} \quad \text { and } \quad L=C Z^{p} K^{3-p}(q+1)^{p-1}(q+2)^{p} .
$$

Our results show that the case $p=2$ plays a critical role and this corresponds to $\lim _{s \rightarrow 0} S(s) / s^{3}=K \in(0, \infty)$, where $L=100 C Z^{2} K$, in the case of a column with geometrically similar cross-sections. In the case where $S(z)$ is constant (equivalently, $A(t)=t^{1 / 2}$ ), equation (1.7) was derived by Bernoulli [2] in the same paper as his original proposition of the Bernoulli-Euler law for the bending moment (see equation (90) in Truesdell's authoritative commentaries [18]).

Special cases of the linear equation (1.1) have been discussed in some situations where the solutions can be expressed in terms of Bessel functions. For a cylindrical column, Euler himself dealt with the case $S(z) \equiv 1$ (equivalently, $A(t)=t^{1 / 2}$ ) $[6,7]$, and this work is discussed at length by Truesdell (see his equation (424) with $P=0$ in [18, pp. 358-367]). This case is also treated by Love [12, p. 425]. Greenhill [8] treats the cases $S(z) \equiv 1-z$ (the set $B$ is a paraboloid of revolution, equivalently $A(t)=t^{4 / 3}$ ) and $S(z)=(1-z)^{2}$ (the set $B$ is a right circular cone, equivalently $\left.A(t)=t^{7 / 4}\right)$, but pays little attention to the boundary conditions at $s=0$. However, he does make some quaint interpretations of his results to structures like pine 
trees and jellies. Further work on special cases of this kind is reported in [17]. The fact that $\mu=\frac{1}{4} L$ is the infimum of the essential spectrum of (1.1)-(1.3) when $\lim _{t \rightarrow 0} A(t) / t^{2}=L$ (equivalently, $\xi=25 K$ when $\lim _{s \rightarrow 0} S(s) / s^{3}=K$ ) seems to have been observed first by Cox and McCarthy [4]. This leads them to criticize previous work on the existence and shape of a column of greatest height [11], which predicts that such a column will have a profile with tapering of order 2 . Note that all these works deal with the cases where the column has geometrically similar cross-sections.

\subsection{Mathematical problem and results}

In this section we discuss the spectral theory of the problem (1.1), (1.2) and (1.3) and summarize our main results. Let $A$ be a profile with tapering of order $p$. For such a profile, there exist constants $K_{1} \geqslant K_{2}>0$ such that

$$
K_{2} s^{p} \leqslant A(s) \leqslant K_{1} s^{p} \text { for all } s \in[0,1] .
$$

Definition 1.2. Since $A$ need not be differentiable, we stipulate that a solution of (1.1)-(1.3) is a function $u \in C^{1}((0,1])$ with $A u^{\prime} \in C^{1}((0,1])$,

$$
\begin{aligned}
\left\{A(s) u^{\prime}(s)\right\}^{\prime}+\mu u(s) & =0 \quad \text { for all } s \in(0,1], \\
u(1) & =\lim _{s \rightarrow 0} A(s) u^{\prime}(s)=0
\end{aligned}
$$

and

$$
\int_{0}^{1} A(s) u^{\prime}(s)^{2} \mathrm{~d} s<\infty .
$$

If $u \not \equiv 0$, it will be called an eigenfunction associated with the eigenvalue $\mu$.

A rigorous discussion of the spectral theory of (1.1)-(1.3) must be formulated in the context of appropriate function spaces and there are two natural (and as we show later, equivalent) ways of doing this.

The first approach is based on using (1.3) to define a natural Hilbert space structure $\left(H_{A},\langle\cdot, \cdot\rangle_{A}\right)$ associated with $A$ and then on studying a bounded selfadjoint operator acting in $H_{A}$. The second method is to introduce a self-adjoint operator in $L^{2}(0,1)$ associated with $(1.1)$ and (1.2). The first alternative is more appropriate for discussing the full nonlinear problems in which (1.1) is replaced by (1.5) or (1.14). Indeed, the space $H_{A}$ consists of all admissible configurations with finite energy and so one can seek the solution with least energy by minimizing the energy in this space. Therefore, $\left(H_{A},\langle\cdot, \cdot\rangle_{A}\right)$, which will be called the energy space, is the best setting for dealing with the nonlinear problem and its rigorous linearization plays a crucial role in its analysis (see $[15,16]$ ). One of the main purposes of the present paper is to establish the essential properties of the linearized problem that are required in the discussion of the nonlinear problem. On the other hand, if we put aside the underlying physical problem, it is standard practice to associate an unbounded linear operator acting in $L^{2}(0,1)$ with a boundary-value problem like (1.1) with (1.2). Here, condition (1.3) plays a subsidiary role, but nonetheless the resulting spectral theory is equivalent to that developed in $H_{A}$. 
For a profile $A$ with tapering of order $p$, the energy space is defined by

$$
H_{A}=\left\{u \in L_{\mathrm{loc}}^{1}((0,1]): \int_{0}^{1} A(s) u^{\prime}(s)^{2} \mathrm{~d} s<\infty \text { and } u(1)=0\right\},
$$

with

$$
\langle u, v\rangle_{A}=\int_{0}^{1} A(s) u^{\prime}(s) v^{\prime}(s) \mathrm{d} s \text { and }\|u\|_{A}=\left\{\int_{0}^{1} A(s) u^{\prime}(s)^{2} \mathrm{~d} s\right\}^{1 / 2} .
$$

Then, for $p \in[0,2]$, a bounded positive self-adjoint operator $T: H_{A} \rightarrow H_{A}$ is defined by

$$
\langle T(u), v\rangle_{A}=\langle u, v\rangle \quad \text { for all } u, v \in H_{A},
$$

where $\langle\cdot, \cdot\rangle$ is the usual scalar product on $L^{2}(0,1)$. It turns out that the eigenfunctions of $T$ are precisely the eigenfunctions of (1.1)-(1.3) defined above. Moreover, it is this operator $T$ that occurs in the analysis of the nonlinear problems concerning the buckling of rods. Therefore, most of our attention is devoted to the spectrum $\sigma(T)$ and essential spectrum $\sigma_{\mathrm{e}}(T)$ of $T$. The alternative point of view is to consider the unbounded self-adjoint operator $N: D(N) \subset L^{2}(0,1) \rightarrow L^{2}(0,1)$ defined by

$$
D(N)=\left\{u \in L^{2}(0,1):\left(A u^{\prime}\right)^{\prime} \in L^{2}(0,1), \text { with } \lim _{s \rightarrow 0} A(s) u^{\prime}(s)=u(1)=0\right\}
$$

and

$$
N(u)=-\left(A u^{\prime}\right)^{\prime} \quad \text { for all } u \in D(N) .
$$

We show in $\S 6$ that $0 \notin \sigma(N)$,

$$
\sigma(N)=\{1 / \lambda: \lambda \in \sigma(T) \backslash\{0\}\} \quad \text { and } \quad \sigma_{\mathrm{e}}(N)=\left\{1 / \lambda: \lambda \in \sigma_{\mathrm{e}}(T) \backslash\{0\}\right\} .
$$

Let us now summarize our conclusions about the spectrum of $T$. For $0 \leqslant p<2$, $T: H_{A} \rightarrow H_{A}$ is a compact operator and 0 is not an eigenvalue of $T$. Furthermore, all eigenvalues of $T$ are simple, so the spectrum can be expressed as

$$
\sigma(T)=\left\{\lambda_{i}: i \in \mathbb{N}\right\} \cup\{0\},
$$

where $\mathbb{N}=\{1,2 \ldots\}, 0<\lambda_{i+1}<\lambda_{i}$ and $\lim _{i \rightarrow \infty} \lambda_{i}=0$. An eigenfunction $\varphi_{i}$ associated with $\lambda_{i}$ has exactly $i$ zeros in $[0,1]$. Also, $\varphi_{i} \in C([0,1])$ and $\varphi_{i}(0) \neq 0$. Thus, for $0 \leqslant p<2$, the spectrum of $T$ resembles that of Green's function for a regular Sturm-Liouville problem. For $p=2, T: H_{A} \rightarrow H_{A}$ is not a compact operator and $\max \sigma_{\mathrm{e}}(T)=4 / L$, where $L=\lim _{s \rightarrow 0} A(s) / s^{2}$. In this case, $T$ may or may not have some eigenvalues in the interval $(4 / L, \infty)$ depending upon the behaviour of $A$ on $(0,1]$. We provide some criteria for settling this question. The existence of at least one eigenvalue (equal to $\|T\|$ ) is a consequence of conditions we give on $A$, which ensure that $\|T\|>4 / L$. In the other direction, we also give properties of $A$ that imply that $T$ has no eigenvalues at all.

The paper is organized as follows. In $\S 2$ we present the fundamental properties of the energy space $H_{A}$ and then in $\S 3$ we introduce the operator $T$. Sections 4 and 5 are devoted to the spectral theory of $T$ in the cases $0 \leqslant p<2$ and $p=2$, respectively. Finally, in $\S 6$ we discuss the differential operator $N$. 


\section{The energy space $H_{A}$}

Consider $p \in[0, \infty)$. If an element $u \in L_{\text {loc }}^{1}((0,1])$ admits a generalized derivative $u^{\prime}$ on $(0,1)$ such that $\int_{0}^{1} s^{p} u^{\prime}(s)^{2} \mathrm{~d} s<\infty$, it follows that $u \in W^{1,1}((\varepsilon, 1))$ for all $\varepsilon \in(0,1)$, and hence, from theorem VIII.2 of [3], that (after modification on a set of measure zero) $u \in C((0,1])$.

For $p \geqslant 0$, let

$$
H_{p}=\left\{u \in L_{\mathrm{loc}}^{1}((0,1]): \int_{0}^{1} s^{p} u^{\prime}(s)^{2} \mathrm{~d} s<\infty \text { and } u(1)=0\right\},
$$

with

$$
\|u\|_{p}=\left\{\int_{0}^{1} s^{p} u^{\prime}(s)^{2} \mathrm{~d} s\right\}^{1 / 2} .
$$

Clearly, $\|\cdot\|_{p}$ is a norm on the linear space $H_{p}$ and

$$
u(x)=-\int_{x}^{1} u^{\prime}(s) \mathrm{d} s
$$

for all $u \in H_{p}$ and all $x \in(0,1]$. Hence, for $u \in H_{p}$ and $x \in(0,1]$,

$$
|u(x)| \leqslant\|u\|_{p}\left\{\int_{x}^{1} s^{-p} \mathrm{~d} s\right\}^{1 / 2},
$$

and so

$$
|u(x)| \leqslant\|u\|_{p}\left\{\frac{1-x^{1-p}}{1-p}\right\}^{1 / 2} \quad \text { if } p \neq 1,
$$

whereas

$$
|u(x)| \leqslant\|u\|_{p}\left\{\ln \frac{1}{x}\right\}^{1 / 2} \quad \text { if } p=1 .
$$

Similarly, for $u \in H_{p}$ and $x, y \in(0,1]$,

$$
|u(x)-u(y)| \leqslant\|u\|_{p}\left\{\left|\int_{x}^{y} s^{-p} \mathrm{~d} s\right|\right\}^{1 / 2} .
$$

Proposition 2.1.

(i) For $p \in[0, \infty), H_{p}$ with the scalar product

$$
\langle u, v\rangle_{p}=\int_{0}^{1} s^{p} u^{\prime}(s) v^{\prime}(s) \mathrm{d} s
$$

is a Hilbert space.

(ii) For any bounded sequence $\left\{u_{n}\right\}$ in $H_{p}$, there exist a function $u \in C((0,1])$ and a subsequence $\left\{u_{n_{k}}\right\}$ such that $u_{n_{k}} \rightarrow u$ uniformly on $[\varepsilon, 1]$ for every $\varepsilon \in(0,1)$.

(iii) $H_{p} \cap L^{\infty}(0,1)$ is dense in $H_{p}$.

(iv) If $u \in H_{p}$, then so does $|u|$ and $|u|^{\prime}(s)^{2}=u^{\prime}(s)^{2}$ almost everywhere on $(0,1)$. 
REMARK 2.2. If $A$ is a profile with tapering of order $p$, then

$$
\langle u, v\rangle_{A}=\int_{0}^{1} A(s) u^{\prime}(s) v^{\prime}(s) \mathrm{d} s
$$

is a scalar product on $H_{A}=H_{p}$ whose norm is equivalent to $\|\cdot\|_{p}$. Indeed,

$$
\sqrt{K_{2}}\|u\|_{p} \leqslant\|u\|_{A} \leqslant \sqrt{K_{1}}\|u\|_{p}
$$

with the constants given in (1.17). The Hilbert space $\left(H_{A},\langle\cdot, \cdot\rangle_{A}\right)$ will be referred to as the energy space for the profile $A$. If the sequence $\left\{u_{n}\right\}$ converges weakly to $u$ in $H_{A}$, then $u_{n} \rightarrow u$ uniformly on $[\varepsilon, 1]$ for every $\varepsilon \in(0,1)$.

REMARK 2.3. Denoting by $A C_{\text {loc }}((0,1])$ the set of all functions that are absolutely continuous on $[\varepsilon, 1]$ for every $\varepsilon \in(0,1)$, the space $H_{A}$ can be characterized as

$$
\left\{u \in A C_{\mathrm{loc}}((0,1]): u(1)=0 \text { and } \int_{0}^{1} A(s) u^{\prime}(s)^{2} \mathrm{~d} s<\infty\right\} .
$$

REMARK 2.4. Setting

$$
u_{\alpha}(s)=s^{\alpha}(1-s) \text { for } 0<s \leqslant 1 \text {, }
$$

we see that $u_{\alpha} \in H_{p} \Longleftrightarrow \alpha>\frac{1}{2}(1-p)$. Noting that the function $\ln \{\ln \mathrm{e} / s\}$ belongs to $H_{1}$ and recalling $(2.1),(2.2)$, we see that $H_{p} \subset L^{\infty}(0,1) \Longleftrightarrow p<1$.

Proof. (i) Consider a Cauchy sequence $\left\{u_{n}\right\}$ in $H_{p}$. Clearly, $\left\{u_{n}^{\prime}\right\}$ is a Cauchy sequence in $L^{2}(\delta, 1)$ for every $\delta \in(0,1)$, and so there is an element $v \in L_{\text {loc }}^{2}((0,1])$ such that

$$
\int_{\delta}^{1}\left\{u_{n}^{\prime}-v\right\}^{2} \mathrm{~d} x \rightarrow 0 \quad \text { as } n \rightarrow \infty \quad \text { for all } \delta \in(0,1) .
$$

Also, for any $\delta \in(0,1)$, it follows from $(2.3)$ that $\left\{u_{n}\right\}$ is a Cauchy sequence in $C([\delta, 1])$. It follows easily that there is a function $u \in C((0,1])$ such that $u_{n} \rightarrow u$ uniformly on $[\delta, 1]$ for every $\delta \in(0,1)$. For any function $w \in C_{0}^{\infty}((0,1))$,

$$
\int_{0}^{1} u w^{\prime} \mathrm{d} x=\lim _{n \rightarrow \infty} \int_{0}^{1} u_{n} w^{\prime} \mathrm{d} x=-\lim _{n \rightarrow \infty} \int_{0}^{1} u_{n}^{\prime} w \mathrm{~d} x=-\int_{0}^{1} v w \mathrm{~d} x
$$

showing that $v$ is the generalized derivative of $u$ on $(0,1)$. Furthermore, for $\delta \in(0,1)$,

$$
\int_{\delta}^{1} s^{p} v(s)^{2} \mathrm{~d} s=\lim _{n \rightarrow \infty} \int_{\delta}^{1} s^{p} u_{n}^{\prime}(s)^{2} \mathrm{~d} s \leqslant \sup _{n}\left\|u_{n}\right\|_{p}^{2}<\infty
$$

and so $u \in H_{p}$.

Choosing $\varepsilon>0$, there exists $k$ such that $\left\|u_{n}-u_{m}\right\|_{p}<\varepsilon$ for all $n, m \geqslant k$. Also, there exists $\delta \in(0,1)$ such that

$$
\int_{0}^{\delta} s^{p} v(s)^{2} \mathrm{~d} s+\int_{0}^{\delta} s^{p} u_{k}^{\prime}(s)^{2} \mathrm{~d} s<\varepsilon,
$$


and hence

$$
\begin{aligned}
& \int_{0}^{1} s^{p}\left\{u_{n}^{\prime}(s)-v(s)\right\}^{2} \mathrm{~d} s \\
& \leqslant 2 \int_{0}^{\delta} s^{p} v(s)^{2} \mathrm{~d} s+2 \int_{0}^{\delta} s^{p} u_{n}^{\prime}(s)^{2} \mathrm{~d} s+\int_{\delta}^{1} s^{p}\left\{u_{n}^{\prime}(s)-v(s)\right\}^{2} \mathrm{~d} s \\
& \leqslant 2 \int_{0}^{\delta} s^{p} v(s)^{2} \mathrm{~d} s+4 \int_{0}^{\delta} s^{p} u_{k}^{\prime}(s)^{2} \mathrm{~d} s \\
& \quad+4\left\|u_{n}-u_{k}\right\|_{p}^{2}+\int_{\delta}^{1} s^{p}\left\{u_{n}^{\prime}(s)-v(s)\right\}^{2} \mathrm{~d} s \\
& \leqslant 4 \varepsilon+4 \varepsilon+\int_{\delta}^{1}\left\{u_{n}^{\prime}(s)-v(s)\right\}^{2} \mathrm{~d} s \quad \text { for all } n \geqslant k .
\end{aligned}
$$

It follows that

$$
\left\|u_{n}-u\right\|_{p}^{2}=\int_{0}^{1} s^{p}\left\{u_{n}^{\prime}(s)-v(s)\right\}^{2} \mathrm{~d} s \rightarrow 0,
$$

showing that $H_{p}$ is complete.

(ii) Let $\left\{u_{n}\right\}$ be a bounded sequence in $H_{p}$ and fix $\delta \in(0,1)$. By $(2.1)-(2.3),\left\{u_{n}\right\}$ is uniformly bounded and equicontinuous on $[\delta, 1]$. The conclusion follows from the theorem of Ascoli and Arzela and a standard diagonalization procedure.

(iii) For $u \in H_{p}$ and $n \in \mathbb{N}$, set

$$
u_{n}(s)= \begin{cases}u(s) & \text { for } 1 / n \leqslant s \leqslant 1 \\ u(1 / n) & \text { for } 0 \leqslant s<1 / n\end{cases}
$$

Then $u_{n} \in H_{p} \cap L^{\infty}(0,1)$ and

$$
\left\|u_{n}-u\right\|_{p}^{2}=\int_{0}^{1 / n} s^{p} u^{\prime}(s)^{2} \mathrm{~d} s \rightarrow 0 \quad \text { as } n \rightarrow \infty .
$$

(iv) See corollaries 20.12 and 20.13 of [10], for example.

By (2.1), $H_{p} \subset L^{2}(0,1)$ for $p<2$. The following result is in the spirit of Hardy's inequality (see p. 327 of [9]), and shows that $H_{p} \subset L^{2}(0,1)$ for $p \leqslant 2$. For $p>2$ and $\alpha \in\left(\frac{1}{2}(1-p),-\frac{1}{2}\right]$ the function $u_{\alpha}$ defined by $(2.5)$ belongs to $H_{p}$, but not to $L^{2}(0,1)$.

The usual scalar product and norm on $L^{2}(0,1)$ are denoted by $\langle\cdot, \cdot\rangle$ and $|\cdot|_{2}$, respectively.

LemmA 2.5. Let $0 \leqslant p \leqslant 2$. Then $H_{p} \subset L^{2}(0,1)$ and

$$
|u|_{2} \leqslant 2\|u\|_{p}
$$

for all $u \in H_{p}$. 
Proof. Choose $u \in H_{p}$ and $\varepsilon \in(0,1)$. Then

$$
\begin{aligned}
\int_{\varepsilon}^{1} u(s)^{2} \mathrm{~d} s & =-\varepsilon u(\varepsilon)^{2}-2 \int_{\varepsilon}^{1} s u(s) u^{\prime}(s) \mathrm{d} s \\
& \leqslant 2\left\{\int_{\varepsilon}^{1} s^{p} u^{\prime}(s)^{2} \mathrm{~d} s\right\}^{1 / 2}\left\{\int_{\varepsilon}^{1} s^{2-p} u(s)^{2} \mathrm{~d} s\right\}^{1 / 2} \\
& \leqslant 2\|u\|_{p}\left\{\int_{\varepsilon}^{1} u(s)^{2} \mathrm{~d} s\right\}^{1 / 2}
\end{aligned}
$$

since $0 \leqslant p \leqslant 2$. This proves $(2.6)$.

\section{The operator $T: H_{A} \rightarrow H_{A}$}

In this section we establish the basic properties of the operator $T$ in $H_{A}$ associated with a profile $A$.

Proposition 3.1. Let $A$ be a profile with tapering of order $p \in[0,2]$. There is a unique bounded linear operator $T: H_{A} \rightarrow H_{A}$ such that

$$
\langle T(u), v\rangle_{A}=\langle u, v\rangle \quad \text { for all } u, v \in H_{A},
$$

where $\langle\cdot, \cdot\rangle$ denotes the usual scalar product on $L^{2}(0,1)$. Furthermore, $T$ is a positive self-adjoint operator in $H_{A}$ and 0 is not an eigenvalue of $T$.

Proof. By lemma 2.5 and $(2.4),\langle\cdot, \cdot\rangle: H_{A} \times H_{A} \rightarrow \mathbb{R}$ is a bounded symmetric bilinear form on $H_{A}$. The existence, uniqueness and self-adjointness of $T$ follow immediately from this via the Riesz representation theorem. Since $\langle T(u), u\rangle_{A}=\langle u, u\rangle>0$ for all $u \in H_{A} \backslash\{0\}$, we see that $T$ is positive and that 0 cannot be an eigenvalue of $T$.

The spectrum of $T$ is the set

$$
\sigma(T)=\left\{\lambda \in \mathbb{R}: T-\lambda I: H_{A} \rightarrow H_{A} \text { is not an isomorphism }\right\} .
$$

Recall (see theorem 1.6 of [5], for example) also that the discrete spectrum of $T$ is the set

$$
\sigma_{\mathrm{d}}(T)=\left\{\lambda \in \sigma(T): T-\lambda I: H_{A} \rightarrow H_{A} \text { is a Fredholm operator }\right\}
$$

and the essential spectrum is its complement,

$$
\sigma_{\mathrm{e}}(T)=\sigma(T) \backslash \sigma_{\mathrm{d}}(T) .
$$

It can be shown (see [5], for example) that $\sigma_{\mathrm{d}}(T)$ is formed by the isolated eigenvalues of $T$ that have finite multiplicity.

Since $T$ is positive and self-adjoint, we know that $\sigma(T) \subset[0, \infty)$ and

$$
\begin{aligned}
\|T\| & =\max \sigma(T) \\
& =\sup \left\{\langle T(u), u\rangle_{A}: u \in H_{A}, \text { with }\|u\|_{A}=1\right\} \\
& =\sup \left\{\frac{\langle u, u\rangle}{\langle u, u\rangle_{A}}: u \in H_{A} \backslash\{0\}\right\} .
\end{aligned}
$$


This can be expressed directly using the Rayleigh quotient for (1.1),

$$
Q_{A}(u)=\frac{\int_{0}^{1} A(s) u^{\prime}(s)^{2} \mathrm{~d} s}{\int_{0}^{1} u(s)^{2} \mathrm{~d} s}
$$

and its infimum

$$
\Lambda(A)=\inf \left\{Q_{A}(u): u \in H_{A} \backslash\{0\}\right\}
$$

Note that since $p \in[0,2]$, it follows from lemma 2.5 that

$$
\Lambda(A) \geqslant \frac{1}{4} K_{2}>0
$$

Hence

$$
\|T\|=\max \sigma(T)=\frac{1}{\Lambda(A)} .
$$

Let us now give an upper bound for $\Lambda(A)$ and sharpen the lower bound (3.1) in a way that will be useful later. These results are based on the following quantity, which is defined for any profile $A$ with tapering of order $p$, where $p \leqslant 2$. For $0<s \leqslant 1$, we set

$$
f_{A}(s)=\frac{4 s^{2}}{A(s)}
$$

and observe that $f_{A} \in C((0,1])$, with

$$
\lim _{s \rightarrow 0} f_{A}(s)= \begin{cases}0 & \text { if } p<2 \\ \frac{4}{L} & \text { if } p=2 .\end{cases}
$$

Now set

$$
M(A)=\sup _{0<s \leqslant 1} f_{A}(s)
$$

THEOREM 3.2. Let $A$ be a profile with tapering of order $p \leqslant 2$. Then $\Lambda(A) \geqslant$ $1 / M(A)$. Furthermore, for any interval $I=[\delta, \gamma] \subset(0,1]$, we have

$$
\Lambda(A) \leqslant \frac{\pi^{2} \max _{s \in I} A(s)}{4|I|\{2 \delta+|I|\}}, \text { where }|I|=\gamma-\delta .
$$

Thus

$$
\frac{4|I|\{2 \delta+|I|\}}{\pi^{2} \max _{s \in I} A(s)} \leqslant\|T\| \leqslant M(A)
$$


Proof. For any $u \in H_{A}$ and any $\varepsilon>0$, we have that

$$
\begin{aligned}
\int_{\varepsilon}^{1} u(s)^{2} \mathrm{~d} s & =-\varepsilon u(\varepsilon)^{2}-2 \int_{\varepsilon}^{1} s u(s) u^{\prime}(s) \mathrm{d} s \\
& \leqslant 2 \int_{\varepsilon}^{1} s\left|u(s) u^{\prime}(s)\right| \mathrm{d} s \\
& \leqslant 2\left\{\int_{\varepsilon}^{1} u(s)^{2} \mathrm{~d} s\right\}^{1 / 2}\left\{\int_{\varepsilon}^{1} s^{2} u^{\prime}(s)^{2} \mathrm{~d} s\right\}^{1 / 2} \\
& =\left\{\int_{\varepsilon}^{1} u(s)^{2} \mathrm{~d} s\right\}^{1 / 2}\left\{\int_{\varepsilon}^{1} f_{A}(s) A(s) u^{\prime}(s)^{2} \mathrm{~d} s\right\}^{1 / 2} \\
& \leqslant\left\{\int_{\varepsilon}^{1} u(s)^{2} \mathrm{~d} s\right\}^{1 / 2}\left\{M(A) \int_{\varepsilon}^{1} A(s) u^{\prime}(s)^{2} \mathrm{~d} s\right\}^{1 / 2}
\end{aligned}
$$

showing that

$$
\int_{\varepsilon}^{1} u(s)^{2} \mathrm{~d} s \leqslant M(A)\|u\|_{A}^{2} .
$$

It follows that

$$
Q_{A}(u) \geqslant \frac{1}{M(A)} \quad \text { for all } u \in H_{A} \backslash\{0\},
$$

and hence $\Lambda(A) \geqslant 1 / M(A)$.

To obtain the upper bound, we fix $0<\delta<\gamma \leqslant 1$ and we construct an element of $H_{A}$ as follows:

$$
u(s)= \begin{cases}1 & \text { for } 0 \leqslant s \leqslant \delta \\ \sin \frac{(\gamma-s) \pi}{2(\gamma-\delta)} & \text { for } \delta<s<\gamma \\ 0 & \text { for } \gamma \leqslant s \leqslant 1\end{cases}
$$

Clearly, $u \in H_{A}$, with

$$
\begin{aligned}
\|u\|_{A}^{2} & =\int_{\delta}^{\gamma} A(s)\left[\frac{\pi}{2(\gamma-\delta)}\right]^{2} \cos ^{2} \frac{(\gamma-s) \pi}{2(\gamma-\delta)} \mathrm{d} s \\
& \leqslant \max _{s \in I} A(s)\left\{\frac{\pi}{2(\gamma-\delta)}\right\}^{2} \int_{\delta}^{\gamma} \cos ^{2} \frac{(\gamma-s) \pi}{2(\gamma-\delta)} \mathrm{d} s \\
& =\frac{\pi^{2}}{8|I|} \max _{s \in I} A(s)
\end{aligned}
$$

and

$$
\int_{0}^{1} u(s)^{2} \mathrm{~d} s=\delta+\int_{\delta}^{\gamma} u(s)^{2} \mathrm{~d} s=\delta+\frac{1}{2}|I| .
$$

Hence

$$
\Lambda(A) \leqslant Q_{A}(u) \leqslant \frac{\pi^{2} \max _{s \in I} A(s)}{4|I|\{2 \delta+|I|\}},
$$

as required. 
Finally, we show that the eigenfunctions of problem (1.1)-(1.3) are precisely the eigenfunctions of the operator $T: H_{A} \rightarrow H_{A}$ introduced in proposition 3.1.

LEMmA 3.3. Let $A$ be a profile with tapering of order $p \in[0,2]$. Then $u$ is an eigenfunction of problem (1.1)-(1.3) if and only if $u \in H_{A}$ and $u=\mu T u$. Furthermore, all eigenvalues of $T$ are simple.

Proof. Suppose that $u$ is a solution of (1.1)-(1.3). Then $u \in H_{p}$ and, for all $v \in H_{p}$,

$$
\begin{aligned}
\int_{0}^{1} A(s) u^{\prime}(s) v^{\prime}(s) \mathrm{d} s & =\lim _{\varepsilon \rightarrow 0+} \int_{\varepsilon}^{1} A(s) u^{\prime}(s) v^{\prime}(s) \mathrm{d} s \\
& =-\lim _{\varepsilon \rightarrow 0+}\left\{A(\varepsilon) u^{\prime}(\varepsilon) v(\varepsilon)+\int_{\varepsilon}^{1}\left\{A(s) u^{\prime}(s)\right\}^{\prime} v(s) \mathrm{d} s\right\} \\
& =\int_{0}^{1} \mu u(s) v(s) \mathrm{d} s-\lim _{\varepsilon \rightarrow 0+} A(\varepsilon) u^{\prime}(\varepsilon) v(\varepsilon) \\
& =\mu\langle T u, v\rangle_{A}-\lim _{\varepsilon \rightarrow 0+} A(\varepsilon) u^{\prime}(\varepsilon) v(\varepsilon),
\end{aligned}
$$

since $u v$ is integrable on $(0,1)$ by lemma 2.5 . Thus

$$
\lim _{\varepsilon \rightarrow 0+} A(\varepsilon) u^{\prime}(\varepsilon) v(\varepsilon)=\mu\langle T u, v\rangle_{A}-\langle u, v\rangle_{A}=l \in \mathbb{R} .
$$

Since $u$ is a solution of $(1.1)-(1.3)$,

$$
A(\varepsilon) u^{\prime}(\varepsilon)=-\mu \int_{0}^{\varepsilon} u(s) \mathrm{d} s,
$$

and so by (2.1) and (2.2), there is a constant $C$ such that

$$
\left|A(\varepsilon) u^{\prime}(\varepsilon)\right| \leqslant C \varepsilon^{1 / 2} \quad \text { for all } \varepsilon \in(0,1] .
$$

Using (2.1) and (2.2) to estimate $v$, it follows that $l=0$ for $p<2$. In the case $p=2$, we obtain the same conclusion, since, if $l \neq 0$, it follows that

$$
s^{-1} A(s) u^{\prime}(s) v(s) \notin L^{1}(0,1),
$$

whereas

$$
\begin{aligned}
\int_{0}^{1}\left|s^{-1} A(s) u^{\prime}(s) v(s)\right| \mathrm{d} s & \leqslant\left\{\int_{0}^{1} A(s) u^{\prime}(s)^{2} \mathrm{~d} s\right\}^{1 / 2}\left\{\int_{0}^{1} s^{-2} A(s) v(s)^{2} \mathrm{~d} s\right\}^{1 / 2} \\
& \leqslant \sqrt{K_{1}}\|u\|_{A}\left\{\int_{0}^{1} v(s)^{2} \mathrm{~d} s\right\}^{1 / 2} \\
& \leqslant 2 \sqrt{K_{1}}\|u\|_{A}\|v\|_{2}<\infty
\end{aligned}
$$

by lemma 2.5 . Hence $l=0$ in all cases and

$$
\mu\langle T u, v\rangle_{A}=\langle u, v\rangle_{A} \quad \text { for all } v \in H_{p}
$$

Thus $u=\mu T u$. 
Conversely, if $u \in H_{A}$ and $u=\mu T u$, it follows that $u \in C((0,1])$, with $u(1)=0$ and

$$
\int_{0}^{1} A(s) u^{\prime}(s) v^{\prime}(s) \mathrm{d} s=\int_{0}^{1} \mu u(s) v(s) \mathrm{d} s
$$

for all $v \in H_{p}$. This implies that $A(s) u^{\prime}(s)$ has a generalized derivative on $(0,1)$ that satisfies $\left\{A(s) u^{\prime}(s)\right\}^{\prime}=-\mu u(s)$ for almost all $s \in(0,1)$. Thus $A u^{\prime} \in C^{1}((0,1])$.

Now let $v \in C^{1}([0,1])$ be such that $v(1)=0$ and $v(s)=1$ for all $s \leqslant \frac{1}{2}$. Clearly, $v \in H_{p}$ and, for any $\varepsilon \in\left(0, \frac{1}{2}\right)$,

$$
\begin{aligned}
A(\varepsilon) u^{\prime}(\varepsilon) & =-\int_{\varepsilon}^{1} A(s) u^{\prime}(s) v^{\prime}(s) \mathrm{d} s-\int_{\varepsilon}^{1}\left\{A(s) u^{\prime}(s)\right\}^{\prime} v(s) \mathrm{d} s \\
& =-\int_{0}^{1} A(s) u^{\prime}(s) v^{\prime}(s) \mathrm{d} s+\mu \int_{\varepsilon}^{1} u(s) v(s) \mathrm{d} s \\
& =-\mu \int_{0}^{\varepsilon} u(s) v(s) \mathrm{d} s \\
& =-\mu \int_{0}^{\varepsilon} u(s) \mathrm{d} s .
\end{aligned}
$$

Hence

$$
\left|A(\varepsilon) u^{\prime}(\varepsilon)\right| \leqslant \mu \int_{0}^{\varepsilon}|u(s)| \mathrm{d} s \quad \text { for } \varepsilon \in\left(0, \frac{1}{2}\right),
$$

and so, by $(2.1)$ and $(2.2)$ with $0 \leqslant p \leqslant 2$, there is a constant $C$ such that

$$
\left|A(\varepsilon) u^{\prime}(\varepsilon)\right| \leqslant C \varepsilon^{1 / 2} \quad \text { for } \varepsilon \in\left(0, \frac{1}{2}\right) .
$$

In particular, $\lim _{s \rightarrow 0} A(s) u^{\prime}(s)=0$ and we have shown that $u$ is a solution of problem $(1.1)-(1.3)$.

If $\lambda$ is a eigenvalue of $T, \lambda \neq 0$ and its eigenfunctions are solutions of (1.1)-(1.3) for $\mu=1 / \lambda$. But the second-order differential equation $(1.1)$ is regular on $(0,1]$ and so the set of solutions satisfying the boundary condition $u(1)=0$ is a onedimensional vector space that contains all the eigenfunctions of $T$ for the value $\lambda$. Hence all the eigenvalues of $T$ are simple.

\section{The spectrum of $T$ when $p<2$}

In this case, problem (1.1)-(1.3) behaves like a regular Sturm-Liouville problem.

THEOREM 4.1. Let $A$ be a profile with tapering of order $p$, where $0 \leqslant p<2$. Then $T: H_{A} \rightarrow H_{A}$ is a compact linear operator.

Proof. Let $\left\{u_{n}\right\}$ be a sequence in $H_{A}$ such that $\left\|u_{n}\right\|_{A} \leqslant 1$ for all $n \in \mathbb{N}$. We must show that $\left\{T\left(u_{n}\right)\right\}$ has a convergent subsequence. By passing to a subsequence, we may suppose that $u_{n} \rightarrow u$ weakly in $H_{A}$ for some element $u \in H_{A}$ with $\|u\|_{A} \leqslant 1$. 
Consider any $v \in H_{A}$ and any $\varepsilon \in(0,1)$. Then

$$
\begin{aligned}
\mid\left\langle T\left(u_{n}\right)-\right. & T(u), v\rangle_{A} \mid \\
& =\left|\left\langle u_{n}-u, v\right\rangle\right| \\
& =\left|\int_{0}^{1}\left(u_{n}-u\right) v \mathrm{~d} s\right| \\
& \leqslant \int_{0}^{\varepsilon}\left|u_{n}-u\right||v| \mathrm{d} s+\int_{\varepsilon}^{1}\left|u_{n}-u\right||v| \mathrm{d} s \\
& \leqslant\left\{\int_{0}^{\varepsilon}\left|u_{n}-u\right|^{2} \mathrm{~d} s\right\}^{1 / 2}\left\{\int_{0}^{\varepsilon}|v|^{2} \mathrm{~d} s\right\}^{1 / 2}+\max _{\varepsilon \leqslant s \leqslant 1}\left|\left(u_{n}-u\right)(s)\right| \int_{\varepsilon}^{1}|v| \mathrm{d} s \\
& \leqslant\left|u_{n}-u\right|_{2}\left\{\int_{0}^{\varepsilon}|v|^{2} \mathrm{~d} s\right\}^{1 / 2}+\max _{\varepsilon \leqslant s \leqslant 1}\left|\left(u_{n}-u\right)(s) \| v\right|_{2} \\
& \leqslant 2\left\|u_{n}-u\right\|_{p} S(\varepsilon)\|v\|_{p}+2 \max _{\varepsilon \leqslant s \leqslant 1}\left|\left(u_{n}-u\right)(s)\right|\|v\|_{p},
\end{aligned}
$$

by lemma 2.5 and $(2.1),(2.2)$, where

$$
S(\varepsilon)= \begin{cases}{\left[\int_{0}^{\varepsilon} \frac{1-s^{1-p}}{1-p} \mathrm{~d} s\right]^{1 / 2}} & \text { if } p \neq 1, \\ {\left[\int_{0}^{\varepsilon} \ln \left(\frac{1}{s}\right) \mathrm{d} s\right]^{1 / 2}} & \text { if } p=1 .\end{cases}
$$

Hence

$$
\begin{aligned}
\left\|T\left(u_{n}-u\right)\right\|_{A} & \leqslant \frac{2}{\sqrt{K_{2}}}\left\{\frac{1}{\sqrt{K_{2}}}\left\|u_{n}-u\right\|_{A} S(\varepsilon)+\max _{\varepsilon \leqslant s \leqslant 1}\left|\left(u_{n}-u\right)(s)\right|\right\} \\
& \leqslant \frac{2}{\sqrt{K_{2}}}\left\{\frac{2}{\sqrt{K_{2}}} S(\varepsilon)+\max _{\varepsilon \leqslant s \leqslant 1}\left|\left(u_{n}-u\right)(s)\right|\right\}
\end{aligned}
$$

and so

$$
\lim \sup _{n \rightarrow \infty}\left\|T\left(u_{n}-u\right)\right\|_{A} \leqslant \frac{4 S(\varepsilon)}{K_{2}}
$$

for any $\varepsilon \in(0,1)$, since $\max _{\varepsilon \leqslant s \leqslant 1}\left|\left(u_{n}-u\right)(s)\right| \rightarrow 0$ as $n \rightarrow \infty$ by remark 2.2. But $S(\varepsilon) \rightarrow 0$ as $\varepsilon \rightarrow 0$, since $p<2$, showing that $\lim _{n \rightarrow \infty}\left\|T\left(u_{n}\right)-T(u)\right\|_{A}=0$. Thus $T$ is compact.

TheOREm 4.2. Let $A$ be a profile with tapering of order $p$, where $0 \leqslant p<2$. Then

$$
\sigma_{\mathrm{d}}(T)=\left\{\lambda_{i}: i \in \mathbb{N}\right\} \quad \text { and } \quad \sigma_{\mathrm{e}}(T)=\{0\},
$$

where $\lambda_{i+1}<\lambda_{i}, \lambda_{1}=\Lambda(A)^{-1}, \lim _{i \rightarrow \infty} \lambda_{i}=0$ and each $\lambda_{i}$ is a simple eigenvalue of $T$. If $\varphi_{i}$ is an eigenfunction of $T$ associated with $\lambda_{i}$, then we have the following.

(a) $\varphi_{i} \in C^{1}((0,1]) \cap L^{\infty}(0,1)$.

(b) $\lim _{s \rightarrow 0} \varphi_{i}(s)$ exists. It is finite and non-zero.

(c) $\varphi_{i}$ has exactly $i$ zeros in $(0,1]$ and all the zeros of $\varphi_{i}$ are simple. 
Proof. By proposition 3.1 and theorem 4.1, T: $H_{A} \rightarrow H_{A}$ is a compact positive self-adjoint operator and so $\sigma_{\mathrm{d}}(T)=\sigma(T) \backslash\{0\} \subset(0, \infty)$. Furthermore, 0 is not an eigenvalue of $T$ and, by lemma 3.3, all the eigenvalues of $T$ are simple.

It follows that $\sigma_{\mathrm{d}}(T)$ is a decreasing sequence $\left\{\lambda_{i}: i \in \mathbb{N}\right\}$, with $\lambda_{1}=\|T\|=$ $\Lambda(A)^{-1}$ and $\lim _{i \rightarrow \infty} \lambda_{i}=0$.

We now turn to a more detailed discussion of the eigenfunctions. Let $\varphi$ be an eigenfunction of $T$ associated with an eigenvalue $\lambda$. By lemma 3.3, $\varphi$ is a solution of (1.1)-(1.3), and so, for $s>0$,

$$
\varphi(s)=\frac{1}{\lambda} \int_{s}^{1} A(\sigma)^{-1} \int_{0}^{\sigma} \varphi(t) \mathrm{d} t \mathrm{~d} \sigma
$$

since $\varphi \in L^{1}(0,1)$ by $(2.1)$ and $(2.2)$. Hence, for $0<s \leqslant 1$,

$$
|\varphi(s)| \leqslant \frac{1}{\lambda K_{2}} \int_{s}^{1} \sigma^{-p} \int_{0}^{\sigma}|\varphi(t)| \mathrm{d} t \mathrm{~d} \sigma .
$$

(a) Setting $E=[0,1) \cap\left\{\alpha: s^{\alpha} \varphi(s) \in L^{\infty}(0,1)\right\}$, we intend to prove that $0 \in E$. Since $\varphi \in H_{p}$, we have that $0 \in E$ if $p<1,(0,1) \subset E$ if $p=1$, and $\left(\frac{1}{2}(p-1), 1\right) \subset E$ if $p>1$, by (2.1) and (2.2). Thus $E \neq \emptyset$ and we set $\gamma=\inf E$. If $\alpha \in E$, it follows from (4.2) that there is a constant $C$ such that

$$
|\varphi(s)| \leqslant C \int_{s}^{1} \sigma^{-p} \int_{0}^{\sigma} t^{-\alpha} \mathrm{d} t \mathrm{~d} \sigma=\frac{C}{1-\alpha} \int_{s}^{1} \sigma^{1-p-\alpha} \mathrm{d} \sigma .
$$

If $\gamma \geqslant 2-p$, this implies that $\alpha+p-2 \in E$ for all $\alpha \in E$, with $\alpha>\gamma$. Hence $\alpha+p-2 \geqslant \gamma$ for all $\alpha \in E$, with $\alpha>\gamma$, contradicting the fact that $p<2$. Thus $\gamma<2-p$ and we can choose $\alpha \in E$ such that $\alpha<2-p$. Now (4.3) implies that $0 \in E$ and we have shown that $\varphi \in L^{\infty}(0,1)$. Since $\varphi$ is a solution of (1.1)-(1.3), we also have that $\varphi \in C^{1}((0,1])$.

(b) Setting

$$
Q(s)=\frac{s}{A(s)}
$$

we see that $0 \leqslant Q(s) \leqslant s^{1-p} / K_{2}$, and so $Q \in L^{1}(0,1)$. Since

$$
\left|A(\sigma)^{-1} \int_{0}^{\sigma} \varphi(t) \mathrm{d} t\right| \leqslant \sup _{0<t \leqslant 1}|\varphi(t)| Q(\sigma)
$$

and $\varphi \in L^{\infty}(0,1)$, it follows from (4.1) that

$$
\lim _{s \rightarrow 0} \varphi(s)=\eta, \quad \text { where } \eta \equiv \frac{1}{\lambda} \int_{0}^{1} A(\sigma)^{-1} \int_{0}^{\sigma} \varphi(t) \mathrm{d} t \mathrm{~d} \sigma \in \mathbb{R} .
$$

But then (4.1) can be written as

$$
\varphi(s)=\eta-\frac{1}{\lambda} \int_{0}^{s} A(\sigma)^{-1} \int_{0}^{\sigma} \varphi(t) \mathrm{d} t \mathrm{~d} \sigma
$$

for $s>0$. If $\eta=0$, this implies that, for all $s \in(0,1]$,

$$
|\varphi(s)| \leqslant \frac{1}{\lambda} \max _{0<t \leqslant s}|\varphi(t)| \int_{0}^{s} Q(\sigma) \mathrm{d} \sigma,
$$


from which it follows that

$$
\max _{0<s \leqslant \tau}|\varphi(s)| \leqslant \frac{1}{\lambda} \max _{0<s \leqslant \tau}|\varphi(s)| \int_{0}^{\tau} Q(\sigma) \mathrm{d} \sigma
$$

for $0<\tau \leqslant 1$. But $Q \in L^{1}(0,1)$, and so there must exist $\tau>0$ such that $\max _{0<s \leqslant \tau}|\varphi(s)|=0$. Then $\varphi(\tau)=\varphi^{\prime}(\tau)=0$, and since $\varphi$ satisfies the differential equation (1.1), we conclude that $\varphi \equiv 0$ on $(0,1]$, contradicting the fact that $\varphi$ is an eigenfunction of $T$. Hence $\eta \neq 0$.

(c) Using the fact that $Q \in L^{1}(0,1)$, this can be established in the same way as for regular Sturm-Liouville problems via the Prüfer transformation. In fact, our problem is a special case of exercise XX(c) in [19], where the details are outlined.

\section{The spectrum of $T$ when $p=2$}

The preceding theorem shows that, for $0 \leqslant p<2$, problem (1.1)-(1.3) behaves like a regular Sturm-Liouville problem, in particular, $\sigma_{\mathrm{e}}(T)=\{0\}$. For $p=2$, the situation is different. Then it is always the case that $\max \sigma_{\mathrm{e}}(T)>0$ and it may happen that $\sigma_{\mathrm{d}}(T)=\emptyset$. We now give a series of results that justify these statements in a sharper form.

\subsection{The essential spectrum of $T$}

We deal first with the special case $A(s)=L s^{2}$ for some $L>0$ and then use this as a basis for treating a general profile with tapering of order 2 .

Lemma 5.1. Let $B(s)=L s^{2}$ for some $L>0$. Then $\Lambda(B)=\frac{1}{4} L$. Furthermore, for any $\varepsilon>0$ and any $\delta>0$, there exists an element $u \in H_{B}$ such that

$$
\frac{1}{4} L \leqslant Q_{B}(u)<\frac{1}{4} L+\varepsilon \text { and } u(s)=0 \text { for all } s \geqslant \delta .
$$

Proof. By lemma 2.5, we have that $\Lambda(B) \geqslant \frac{1}{4} L$. Now consider the functions $u_{\alpha}$ defined in (2.5). For all $\alpha>-\frac{1}{2}, u_{\alpha} \in H_{2}$, and, for the profile $B$, the Rayleigh quotient $Q_{B}\left(u_{\alpha}\right)$ can be calculated by elementary integrations. One finds that $Q_{B}\left(u_{\alpha}\right) \rightarrow \frac{1}{4} L$ as $\alpha$ tends to $-\frac{1}{2}$ from above. Thus $\Lambda(B) \leqslant \frac{1}{4} L$, as required.

Furthermore, for this profile, the Rayleigh quotient $Q_{B}$ scales perfectly in the following sense. Given any $u \in H_{2}$ and any $\beta \geqslant 1$, we set

$$
u_{\beta}(s)= \begin{cases}u(\beta s) & \text { for } 0 \leqslant s \leqslant 1 / \beta, \\ 0 & \text { for } 1 / \beta<s \leqslant 1 .\end{cases}
$$

It follows that $u_{\beta} \in H_{2}$ and that $Q_{B}\left(u_{\beta}\right)=Q_{B}(u)$ for all $\beta \geqslant 1$. For any $\varepsilon>0$, there exists an element $u \in H_{2}$ such that $Q_{B}(u)<\frac{1}{4} L+\varepsilon$. Choosing $\beta \geqslant \max \{1,1 / \delta\}$, we see that $Q_{B}\left(u_{\beta}\right)<\frac{1}{4} L+\varepsilon$ and $u_{\beta}(s)=0$ for all $s \geqslant \delta$.

Corollary 5.2. Let $B(s)=L s^{2}$ for some $L>0$ and let $S: H_{B} \rightarrow H_{B}$ be the bounded self-adjoint operator defined by

$$
\langle S u, v\rangle_{B}=\int_{0}^{1} u(s) v(s) \mathrm{d} s \quad \text { for all } u, v \in H_{B},
$$

as in proposition 3.1. Then we have the following conclusions. 
(i) $\|S\|=4 / L$.

(ii) There is a sequence $\left\{u_{n}: n \in \mathbb{N}\right\} \subset H_{B}$ such that

$$
\begin{gathered}
\left\|u_{n}\right\|_{B}=1, \quad u_{n}(s)=0 \quad \text { for all } s \in\left[\frac{1}{n}, 1\right], \\
\quad \frac{4}{L}-\frac{1}{n}<\left\langle S u_{n}, u_{n}\right\rangle_{B} \leqslant \frac{4}{L}, \\
\left\|\left(S-\frac{4}{L} I\right) u_{n}\right\|_{B} \rightarrow 0 \text { as } n \rightarrow \infty .
\end{gathered}
$$

(iii) $S$ has no eigenvalues, so $\sigma_{\mathrm{d}}(S)=\emptyset$ and $\max \sigma_{\mathrm{e}}(S)=4 / L$.

(iv) For $\mu>\frac{1}{4} L$, every solution of the differential equation (1.1) has an infinite number of zeros in $(0,1)$.

Proof. (i) Recalling that

$$
Q_{B}(u)=\frac{\|u\|_{B}^{2}}{\langle S u, u\rangle_{B}} \quad \text { for all } u \in H_{2} \backslash\{0\},
$$

it follows immediately from the preceding lemma that there is a sequence such that $\left\|u_{n}\right\|_{B}=1, u_{n}(s)=0$ for all $s \in[1 / n, 1]$ and

$$
\frac{4}{L}-\frac{1}{n}<\left\langle S u_{n}, u_{n}\right\rangle_{B} \leqslant \frac{4}{L} .
$$

Furthermore, $\|S\|=1 / \Lambda(B)$ by $(3.2)$, showing that $\|S\|=4 / L$. Also,

$$
\begin{aligned}
\left\|\left(S-\frac{4}{L} I\right) u_{n}\right\|_{B}^{2} & =\left\|S u_{n}\right\|_{B}^{2}+\left(\frac{4}{L}\right)^{2}\left\|u_{n}\right\|_{B}^{2}-\frac{8}{L}\left\langle S u_{n}, u_{n}\right\rangle_{B} \\
& \leqslant 2\left(\frac{4}{L}\right)^{2}-\frac{8}{L}\left(\frac{4}{L}-\frac{1}{n}\right)=\frac{8}{n L} .
\end{aligned}
$$

This proves parts (i) and (ii).

(iii) and (iv) For the profile $L s^{2}$, equation (1.1) can be written as

$$
s^{2} u^{\prime \prime}(s)+2 s u^{\prime}(s)+\frac{\mu}{L} u(s)=0,
$$

which is of Euler type and can be transformed to

$$
v^{\prime \prime}(t)-v^{\prime}(t)+\frac{\mu}{L} v(t)=0 \quad \text { on }(0, \infty)
$$

by setting $t=-\ln s$ and $v(t)=u(s)$. The general solution of this equation is

$$
v(t)= \begin{cases}\mathrm{e}^{t / 2}\left\{\alpha \mathrm{e}^{t \sqrt{1 / 4-\mu / L}}+\beta \mathrm{e}^{-t \sqrt{1 / 4-\mu / L}}\right\} & \text { if } \mu<\frac{1}{4} L, \\ \mathrm{e}^{t / 2}\{\alpha+\beta t\} & \text { if } \mu=\frac{1}{4} L, \\ \mathrm{e}^{t / 2}\{\alpha \cos (t \sqrt{\mu / L-1 / 4})+\beta \sin (t \sqrt{\mu / L-1 / 4})\} & \text { if } \mu>\frac{1}{4} L,\end{cases}
$$

where $\alpha$ and $\beta$ are real constants. For $\mu>\frac{1}{4} L$ and any $\alpha, \beta \in \mathbb{R}, v$ has a sequence of zeros $t_{n}$ converging to infinity and so $u$ has a sequence of zeros converging to 0 . This proves part (iv) of the lemma. 
Since $B(s) u^{\prime}(s)=-L \mathrm{e}^{-t} v^{\prime}(t)$, all solutions satisfy the boundary condition $\lim _{s \rightarrow 0} B(s) u^{\prime}(s)=0$. Furthermore,

$$
\int_{0}^{1} B(s) u^{\prime}(s)^{2} \mathrm{~d} s=L \int_{0}^{\infty} \mathrm{e}^{-t} v^{\prime}(t)^{2} \mathrm{~d} t
$$

from which it follows that, for $\mu \geqslant \frac{1}{4} L$, only the solution $u \equiv 0$ has

$$
\int_{0}^{1} B(s) u^{\prime}(s)^{2} \mathrm{~d} s<\infty
$$

whereas, for $\mu<\frac{1}{4} L$, the only solutions having this property are of the form $u(s)=$ $\beta s^{\gamma}$, where

$$
\gamma=\sqrt{\frac{1}{4}-\frac{\mu}{L}}-\frac{1}{2}
$$

and $\beta$ is an arbitrary constant. In the latter case, the boundary condition $u(1)=0$ means that $\beta$ must be 0 . Thus, for all $\mu>0, u \equiv 0$ is the only solution of (1.1)-(1.3), proving part (iii).

REMARK 5.3. The proof of parts (iii) and (iv) brings out the essential difference between the conditions $\lim _{s \rightarrow 0} A(s) u^{\prime}(s)=0$ and $\int_{0}^{1} A(s) u^{\prime}(s)^{2} \mathrm{~d} s<\infty$ when $p=2$. In fact, for $p=2, \lim _{s \rightarrow 0} B(s) u^{\prime}(s)=0$ for all solutions of $(1.1)$, whereas for $\mu \geqslant \frac{1}{4} L$, $\int_{0}^{1} B(s) u^{\prime}(s)^{2} \mathrm{~d} s=\infty$ for all non-trivial solutions. For $p<2$, the situation is completely different, since $\int_{0}^{1} A(s) u^{\prime}(s)^{2} \mathrm{~d} s$ is finite for all solutions of the differential equation (1.1) that satisfy $\lim _{s \rightarrow 0} A(s) u^{\prime}(s)=0$. Indeed, using only (1.1) and $\lim _{s \rightarrow 0} A(s) u^{\prime}(s)=0$ we obtain (3.5), from which it follows that

$$
A(s) u^{\prime}(s)^{2}=A(s)^{-1}\left[A(s) u^{\prime}(s)\right]^{2} \leqslant K_{2}^{-1} s^{-p}\left[C s^{1 / 2}\right]^{2}=\left[C^{2} / K_{2}\right] s^{1-p}
$$

for all $s \in(0,1]$. For $p<2$, this implies that $\int_{0}^{1} A(s) u^{\prime}(s)^{2} \mathrm{~d} s<\infty$.

Having established these results for the profile $B(s)=L s^{2}$, we now return to the discussion of general profiles with tapering of order 2 .

LEMMA 5.4. Let $A$ be a profile with tapering of order 2 . Then $\Lambda(A) \leqslant \frac{1}{4} L$, where $L=\lim _{s \rightarrow 0} A(s) / s^{2}$ and, for $\mu>\frac{1}{4} L$, all solutions of equation (1.1) have infinitely many zeros.

Proof. For $s \in(0,1]$, let $r(s)=A(s) / s^{2}$. For any $u \in H_{2}$ and any $\beta \geqslant 1$, let $u_{\beta}$ be defined by (5.1). Then

$$
Q_{A}\left(u_{\beta}\right)=\frac{\int_{0}^{1 / \beta} r(s) s^{2} u_{\beta}^{\prime}(s)^{2} \mathrm{~d} s}{\int_{0}^{1 / \beta} u_{\beta}(s)^{2} \mathrm{~d} s}=\frac{\int_{0}^{1} r(s / \beta) s^{2} u^{\prime}(s)^{2} \mathrm{~d} s}{\int_{0}^{1} u(s)^{2} \mathrm{~d} s}
$$

and so, setting $B(s)=L s^{2}$ for $s \in[0,1]$, we find that

$$
\lim _{\beta \rightarrow \infty} Q_{A}\left(u_{\beta}\right)=\frac{\int_{0}^{1} L s^{2} u^{\prime}(s)^{2} \mathrm{~d} s}{\int_{0}^{1} u(s)^{2} \mathrm{~d} s}=Q_{B}(u)
$$

by dominated convergence, since $\lim _{s \rightarrow 0} r(s)=L$. It follows that $\Lambda(A) \leqslant \Lambda(B)$. But $\Lambda(B)=\frac{1}{4} L$ by lemma 5.1 . 
Now fix $\mu>\frac{1}{4} L$ and choose $\varepsilon>0$ such that $\mu /(L+\varepsilon)>\frac{1}{4}$. There exists $\delta>0$ such that

$$
(L-\varepsilon) s^{2} \leqslant A(s) \leqslant(L+\varepsilon) s^{2} \quad \text { for all } s \in[0, \delta] .
$$

Consider the equation

$$
\left\{(L+\varepsilon) s^{2} v^{\prime}(s)\right\}^{\prime}+\mu v(s)=0 \quad \text { on }(0, \delta)
$$

and let $v \not \equiv 0$ be a solution. By the Sturm comparison theorem (see lemma 3.2 in ch. II of [13]), every solution of (1.1) has at least as many zeros as $v$ in $(0, \delta)$. But $v$ satisfies

$$
\left\{s^{2} v^{\prime}(s)\right\}^{\prime}+\frac{\mu}{(L+\varepsilon)} v(s)=0 \quad \text { on }(0, \delta),
$$

where $\mu /(L+\varepsilon)>\frac{1}{4}$, so it follows from corollary 5.2 (iv) that $v$ has an infinite number of zeros in $(0, \delta)$. This completes the proof.

Lemma 5.5. Let $A$ be a profile with tapering of order 2 . Then $4 / L \in \sigma_{\mathrm{e}}(T)$, where $L=\lim _{s \rightarrow 0} A(s) / s^{2}$. Furthermore,

$$
\max \sigma_{\mathrm{e}}(T) \leqslant \frac{4}{K_{2}}, \quad \text { where } K_{2}=\inf _{0<s \leqslant 1} \frac{A(s)}{s^{2}} .
$$

REMARK 5.6. Let $A$ be a profile with tapering of order 2. Since $\max \sigma_{\mathrm{e}}(T)>0$, it follows that the self-adjoint operator $T: H_{A} \rightarrow H_{A}$ is not compact.

Proof. Let $\lambda=4 / L$ and let $B(s)=L s^{2}$ for $0 \leqslant s \leqslant 1$. There is a sequence $\left\{\varepsilon_{n}: n \in \mathbb{N}\right\}$ such that $\varepsilon_{n} \rightarrow 0$ and

$$
\left(1-\varepsilon_{n}\right) B(s) \leqslant A(s) \leqslant\left(1+\varepsilon_{n}\right) B(s) \text { for } 0 \leqslant s \leqslant \frac{1}{n} .
$$

Now consider the sequence $\left\{u_{n}\right\}$ given by corollary $5.2(\mathrm{ii})$. Clearly,

$$
\left\|u_{n}\right\|_{A}^{2}=\int_{0}^{1 / n} A(s) u_{n}^{\prime}(s)^{2} \mathrm{~d} s \in\left[1-\varepsilon_{n}, 1+\varepsilon_{n}\right]
$$

and

$$
\left\langle T u_{n}, u_{n}\right\rangle_{A}=\left\langle S u_{n}, u_{n}\right\rangle_{B} \in\left[\lambda-\frac{1}{n}, \lambda\right] .
$$

Furthermore, in the notation of corollary 5.2, for any $v \in H_{2}$,

$$
\left\langle T u_{n}, v\right\rangle_{A}=\left\langle S u_{n}, v\right\rangle_{B}=\int_{0}^{1 / n} u_{n}(s) v(s) \mathrm{d} s
$$

and

$$
\|v\|_{B} \leqslant \sqrt{\frac{L}{K_{2}}}\|v\|_{A}
$$

Also,

$$
\left\langle u_{n}, v\right\rangle_{A}-\left\langle u_{n}, v\right\rangle_{B}=\int_{0}^{1 / n}[A(s)-B(s)] u_{n}^{\prime}(s) v^{\prime}(s) \mathrm{d} s
$$


so

$$
\begin{aligned}
\left|\left\langle u_{n}, v\right\rangle_{A}-\left\langle u_{n}, v\right\rangle_{B}\right| \\
\quad \leqslant\left\{\int_{0}^{1 / n}|A(s)-B(s)| u_{n}^{\prime}(s)^{2} \mathrm{~d} s\right\}^{1 / 2}\left\{\int_{0}^{1 / n}|A(s)-B(s)| v^{\prime}(s)^{2} \mathrm{~d} s\right\}^{1 / 2} \\
\quad \leqslant \varepsilon_{n}\left\|u_{n}\right\|_{B}\|v\|_{B}=\varepsilon_{n}\|v\|_{B} .
\end{aligned}
$$

It follows that

$$
\begin{aligned}
\left|\left\langle(T-\lambda) u_{n}, v\right\rangle_{A}\right| & =\left|\left\langle(S-\lambda) u_{n}, v\right\rangle_{B}+\lambda\left\langle u_{n}, v\right\rangle_{B}-\lambda\left\langle u_{n}, v\right\rangle_{A}\right| \\
& \leqslant\left|\left\langle(S-\lambda) u_{n}, v\right\rangle_{B}\right|+\lambda\left|\left\langle u_{n}, v\right\rangle_{A}-\left\langle u_{n}, v\right\rangle_{B}\right| \\
& \leqslant\left\|(S-\lambda I) u_{n}\right\|_{B}\|v\|_{B}+\lambda \varepsilon_{n}\|v\|_{B},
\end{aligned}
$$

showing that

$$
\left\|(T-\lambda) u_{n}\right\|_{A} \leqslant \sqrt{\frac{L}{K_{2}}}\left\{\left\|(S-\lambda I) u_{n}\right\|_{B}+\lambda \varepsilon_{n}\right\} .
$$

Hence $\left\|(T-\lambda) u_{n}\right\|_{A} \rightarrow 0$ as $n \rightarrow \infty$, since $\varepsilon_{n} \rightarrow 0$ and $\left\|(S-\lambda I) u_{n}\right\|_{B} \rightarrow 0$. Setting $v_{n}=u_{n} /\left\|u_{n}\right\|_{A}$, we see that $\left\|v_{n}\right\|_{A}=1$ for all $n \in \mathbb{N}$ and

$$
\left\|(T-\lambda) v_{n}\right\|_{A}=\frac{\left\|(T-\lambda) u_{n}\right\|_{A}}{\left\|u_{n}\right\|_{A}} \rightarrow 0,
$$

since $\left\|u_{n}\right\|_{A} \rightarrow 1$. Furthermore, for any $v \in H_{A}$,

$$
\begin{aligned}
\left|\left\langle T v_{n}, v\right\rangle_{A}\right| & =\frac{\left|\int_{0}^{1 / n} u_{n} v \mathrm{~d} s\right|}{\left\|u_{n}\right\|_{A}} \\
& \leqslant \frac{\left|u_{n}\right|_{2}}{\left\|u_{n}\right\|_{A}}\left\{\int_{0}^{1 / n} v^{2} \mathrm{~d} s\right\}^{1 / 2} \\
& \leqslant \frac{2}{\sqrt{K_{2}}}\left\{\int_{0}^{1 / n} v^{2} \mathrm{~d} s\right\}^{1 / 2} .
\end{aligned}
$$

Hence $\left\langle T v_{n}, v\right\rangle_{A} \rightarrow 0$ as $n \rightarrow \infty$, since $H_{A} \subset L^{2}(0,1)$ by lemma 2.5 . From this and the fact that $\left\|(T-\lambda) v_{n}\right\|_{A} \rightarrow 0$, it follows easily that $\left\langle v_{n}, v\right\rangle_{A} \rightarrow 0$ as $n \rightarrow \infty$ for any $v \in H_{A}$. This means that $\left\{v_{n}\right\}$ is what is called in [5] a singular sequence for $T$ corresponding to $\lambda$. Hence, by theorems 1.3 and 1.6 in ch. IX of $[5], \lambda=4 / L \in \sigma_{\mathrm{e}}(T)$.

Conversely, suppose that $\xi \in \sigma_{\mathrm{e}}(T)$. Then there is a singular sequence $\left\{w_{n}\right\} \subset$ $H_{A}$ for $T$ corresponding to $\xi$. Thus $\left\|w_{n}\right\|_{A}=1, w_{n} \rightarrow 0$ weakly in $H_{A}$ and $\left\|(T-\xi I) w_{n}\right\|_{A} \rightarrow 0$. It follows from proposition 2.1 that $w_{n}(s) \rightarrow 0$ uniformly on $[1 / k, 1]$ for every $k \in \mathbb{N}$.

First we observe that $\left\langle(T-\xi I) w_{n}, w_{n}\right\rangle_{A} \rightarrow 0$, and so

$$
\int_{0}^{1} w_{n}(s)^{2} \mathrm{~d} s=\left\langle T w_{n}, w_{n}\right\rangle_{A} \rightarrow \xi
$$

However, using corollary 5.2 ,

$$
\int_{0}^{1} w_{n}(s)^{2} \mathrm{~d} s=\left\langle S w_{n}, w_{n}\right\rangle_{B} \leqslant \frac{4}{L}\left\|w_{n}\right\|_{B}^{2} \leqslant \frac{4}{K_{2}}\left\|w_{n}\right\|_{A}^{2}=\frac{4}{K_{2}} .
$$


Recalling (5.4), this proves that $\xi \leqslant 4 / K_{2}$.

We can now establish the main result of this section in full generality.

TheOREM 5.7. Let $A$ be a profile with tapering of order 2 . Then $\max \sigma_{\mathrm{e}}(T)=4 / L$, where $L=\lim _{s \rightarrow 0} A(s) / s^{2}$.

Proof. We begin by showing that $\sigma_{\mathrm{e}}(T)$ depends only on the behaviour of $A$ near $s=0$. This will be done by decomposing the space $H_{A}$ into three mutually orthogonal subspaces. First, we fix $\delta \in(0,1)$ and then we define an element $w$ of $H_{A}$ by setting

$$
w(s)= \begin{cases}-\int_{\delta}^{1} A(t)^{-1} \mathrm{~d} t & \text { for } 0 \leqslant s \leqslant \delta, \\ -\int_{s}^{1} A(t)^{-1} \mathrm{~d} t & \text { for } \delta<s \leqslant 1 .\end{cases}
$$

Let $P: H_{A} \rightarrow H_{A}$ be the orthogonal projection of $H_{A}$ onto $w^{\perp}$ and set $Q=I-P$. Noting that, for any $u \in H_{A}$,

$$
\langle u, w\rangle_{A}=\int_{0}^{1} A(s) u^{\prime}(s) w^{\prime}(s) \mathrm{d} s=\int_{\delta}^{1} A(s) u^{\prime}(s) A(s)^{-1} \mathrm{~d} s=-u(\delta),
$$

we find that

$$
w^{\perp}=\left\{u \in H_{A}: u(\delta)=0\right\} .
$$

Next, for any $v \in w^{\perp}$, we write $v=v_{1}+v_{2}$, where

$$
v_{1}(s)=0 \text { for } \delta \leqslant s \leqslant 1 \text { and } v_{2}(s)=0 \text { for } 0 \leqslant s \leqslant \delta .
$$

Then $v_{1}$ and $v_{2} \in H_{A}$, with $\left\langle v_{1}, v_{2}\right\rangle_{A}=0$. Thus we have an orthogonal decomposition of $H_{A}$ as

$$
H_{A}=\operatorname{span}\{w\} \oplus E_{1} \oplus E_{2}, \quad \text { with } u=\frac{u(\delta)}{w(\delta)} w+(P u)_{1}+(P u)_{2},
$$

and, by an obvious abuse of notation, we can write

$$
E_{1}=\left\{u \in L_{\mathrm{loc}}^{1}((0, \delta]): \int_{0}^{\delta} s^{p} u^{\prime}(s)^{2} \mathrm{~d} s<\infty \text { and } u(\delta)=0\right\}
$$

and

$$
E_{2}=\left\{u \in L^{1}(\delta, 1): \int_{\delta}^{1} s^{p} u^{\prime}(s)^{2} \mathrm{~d} s<\infty \text { and } u(\delta)=u(1)=0\right\}=H_{0}^{1}(\delta, 1)
$$

in the usual notation for Sobolev spaces [3, § VIII.3].

We now use this decomposition to characterize the essential spectrum of $T$. The subspace $w^{\perp}$ is not invariant under $T$, but the subspaces $E_{i}$ are invariant under $P T P$, since, for $v_{i} \in E_{i}$,

$$
\left\langle P T P v_{1}, v_{2}\right\rangle_{A}=\left\langle T v_{1}, v_{2}\right\rangle_{A}=\int_{0}^{1} v_{1} v_{2} \mathrm{~d} s=0 .
$$


Clearly,

$$
T=P T P+Q T+P T Q,
$$

where $Q T+P T Q: H_{A} \rightarrow H_{A}$ is a compact linear operator, since $Q$ has a onedimensional range. By theorem 4.1 in ch. I of [5], this means that $T$ and PTP have the same essential spectrum. But, in the notation of ch. IX.5 of [5], PTP $=R \oplus S$, where $R w=0$ and $S: w^{\perp} \rightarrow w^{\perp}$ satisfies

$$
\langle S u, v\rangle_{A}=\langle T u, v\rangle_{A}=\int_{0}^{1} u(s) v(s) \mathrm{d} s=\sum_{i=1}^{2} \int_{0}^{1} u_{i}(s) v_{i}(s) \mathrm{d} s
$$

for all $u=u_{1}+u_{2}, v=v_{1}+v_{2} \in w^{\perp}=E_{1} \oplus E_{2}$ and $S=S_{1} \oplus S_{2}$, where $S_{i}: E_{i} \rightarrow E_{i}$ is the unique bounded self-adjoint operator satisfying

$$
\left\langle S_{i} u, v\right\rangle_{A}=\langle T u, v\rangle_{A} \quad \text { for all } u, v \in E_{i} .
$$

From the relations (5.2) in ch. IX.5 of [5], we conclude that

$$
\sigma_{\mathrm{e}}(P T P)=\sigma_{\mathrm{e}}(R) \cup \sigma_{\mathrm{e}}\left(S_{1}\right) \cup \sigma_{\mathrm{e}}\left(S_{2}\right),
$$

where $\sigma_{\mathrm{e}}(R)=\emptyset$. We claim that $\sigma_{\mathrm{e}}\left(S_{2}\right)=\{0\}$. In fact, if $\left\{u_{n}\right\}$ is a bounded sequence in $E_{2}$, by passing to a subsequence, we may suppose that $u_{n} \rightarrow u$ uniformly on $[\delta, 1]$ for some element $u \in E_{2}$. Thus, for any $v \in E_{2}$,

$$
\begin{aligned}
\left|\left\langle S_{2}\left(u_{n}-u\right), v\right\rangle_{A}\right| & \leqslant \int_{\delta}^{1}\left|u_{n}(s)-u(s)\right||v(s)| \mathrm{d} s \\
& \leqslant \max _{\delta \leqslant s \leqslant 1}\left|u_{n}(s)-u(s)\right| \max _{\delta \leqslant s \leqslant 1}|v(s)| \\
& \leqslant C(\delta) \max _{\delta \leqslant s \leqslant 1}\left|u_{n}(s)-u(s)\right|\|v\|_{A}
\end{aligned}
$$

for some constant $C(\delta)$ that is given by (2.1). This proves that $\left\|S_{2}\left(u_{n}-u\right)\right\|_{A} \rightarrow 0$ and hence that $S_{2}: E_{2} \rightarrow E_{2}$ is a compact operator, justifying our claim that $\sigma_{\mathrm{e}}\left(S_{2}\right)=\{0\}$.

We now know that

$$
\sigma_{\mathrm{e}}(T)=\sigma_{\mathrm{e}}(P T P)=\{0\} \cup \sigma_{\mathrm{e}}\left(S_{1}\right)
$$

so it remains to investigate the spectrum of $S_{1}: E_{1} \rightarrow E_{1}$. As we shall now show, this is simply a rescaling of $(1.1),(1.2),(1.3)$ to the interval $[0, \delta]$, and so we can use lemma 5.5 to estimate $\max \sigma_{\mathrm{e}}\left(S_{1}\right)$. To do this, we define a new profile $D$ on $[0,1]$ by setting

$$
D(s)=\delta^{-1} A(\delta s) \text { for } 0 \leqslant s \leqslant 1
$$

with

$$
l=\lim _{s \rightarrow 0} \frac{D(s)}{s^{2}} \quad \text { and } \quad k_{2}=\inf _{0<s \leqslant 1} \frac{D(s)}{s^{2}} .
$$

Clearly,

$$
l=\delta L \quad \text { and } \quad k_{2}=\delta \inf _{0<t \leqslant \delta} \frac{A(t)}{t^{2}} .
$$


Furthermore, the operator $J: E_{1} \rightarrow H_{D}$ defined by

$$
J u(t)=u(\delta t) \quad \text { for } 0 \leqslant t \leqslant 1
$$

is an isometric isomorphism of the Hilbert space $\left(E_{1},\langle\cdot, \cdot\rangle_{A}\right)$ onto $\left(H_{D},\langle\cdot, \cdot\rangle_{D}\right)$, since, for any $u, v \in E_{1}$,

$$
\begin{aligned}
\langle u, v\rangle_{A} & =\int_{0}^{\delta} A(s) u^{\prime}(s) v^{\prime}(s) \mathrm{d} s \\
& =\delta \int_{0}^{1} A(\delta t) u^{\prime}(\delta t) v^{\prime}(\delta t) \mathrm{d} t \\
& =\delta^{-1} \int_{0}^{1} A(\delta t)(J u)^{\prime}(t)(J v)^{\prime}(t) \mathrm{d} t \\
& =\int_{0}^{1} D(t)(J u)^{\prime}(t)(J v)^{\prime}(t) \mathrm{d} t \\
& =\langle J u, J v\rangle_{D} .
\end{aligned}
$$

Let $V: H_{D} \rightarrow H_{D}$ be the self-adjoint operator associated with (1.1)-(1.3) for the profile $D$ via proposition 3.1. Then, for all $u, v \in E_{1}$,

$$
\begin{aligned}
\langle V J u, J v\rangle_{D} & =\int_{0}^{1} J u(t) J v(t) \mathrm{d} t \\
& =\delta^{-1} \int_{0}^{\delta} u(s) v(s) \mathrm{d} s \\
& =\delta^{-1}\left\langle S_{1} u, v\right\rangle_{A} \\
& =\delta^{-1}\left\langle J S_{1} u, J v\right\rangle_{D},
\end{aligned}
$$

thus $J^{-1} V J=\delta^{-1} S_{1}$. It follows that $\max \sigma_{\mathrm{e}}\left(S_{1}\right)=\delta \max \sigma_{\mathrm{e}}(V)$ and, by lemma 5.5 applied to the profile $D, \max \sigma_{\mathrm{e}}(V) \in\left[4 / l, 4 / k_{2}\right]$, where $l$ and $k_{2}$ are defined in (5.7). Hence, using (5.8),

$$
\frac{4}{L} \leqslant \max \sigma_{\mathrm{e}}\left(S_{1}\right) \leqslant \frac{4}{\inf _{0<t \leqslant \delta} A(t) / t^{2}}
$$

and $\inf _{0<t \leqslant \delta} A(t) / t^{2} \rightarrow L$ as $\delta \rightarrow 0$. This proves that $\max \sigma_{\mathrm{e}}\left(S_{1}\right)=4 / L$ and, consequently, that $\max \sigma_{\mathrm{e}}(T)=4 / L$ by $(5.6)$.

\subsection{Existence and non-existence of eigenfunctions}

Recalling from (3.2) that $\Lambda(A)=\inf \sigma\left(T^{-1}\right)$, we introduce the notation

$$
\Lambda_{\mathrm{e}}(A)=\inf \sigma_{\mathrm{e}}\left(T^{-1}\right) \text {. }
$$

Combining theorems 3.2 and 5.7, we obtain the following information about the spectrum of (1.1)-(1.3).

TheOREM 5.8. Let $A$ be a profile with tapering of order $p=2$. Then

$$
\frac{1}{M(A)} \leqslant \Lambda(A) \leqslant \frac{1}{4} L=\Lambda_{\mathrm{e}}(A)
$$


where $L=\lim _{s \rightarrow 0} A(s) / s^{2}$ and $M(A)$ is defined by equation (3.4). In particular, $\Lambda(A)=\Lambda_{\mathrm{e}}(A)=\frac{1}{4} L$, provided that

$$
A(s) \geqslant L s^{2} \quad \text { for all } s \in(0,1] \text {. }
$$

REMARK 5.9. To show that $\Lambda(A)<\Lambda_{\mathrm{e}}(A)$, it is enough to find one function $u \in H_{2}$ for which $Q_{A}(u)<\frac{1}{4} L$. On the other hand, theorem 3.2 can be used to exhibit a class of profiles for which this is possible, since it is enough to ensure that

$$
\frac{\pi^{2} \max _{s \in I} A(s)}{|I|\{2 \delta+|I|\}}<L
$$

for some interval $I=[\delta, \gamma] \subset(0,1]$. Recalling Greenhill's interpretation of the problem in connection with pine trees [8], we observe that every profile $A$ can be modified on a small interval $I$ near the clamped end so as to make

$$
\frac{\pi^{2} \max _{s \in I} A(s)}{|I|\{2 \delta+|I|\}}
$$

arbitrarily small. This principle has been used, at least since the time of Noah, to fell trees.

Finally, we show that in some cases there may be no eigenfunctions at all.

TheOREm 5.10. Let $A$ be a profile with tapering of order 2. Suppose that $A \in$ $C^{1}([0,1])$, with $\lim _{s \rightarrow 0} A^{\prime}(s) / s=2 L$, and that, for all $s \in(0,1]$,

$$
\frac{\mathrm{d} h}{\mathrm{~d} s}(s) \leqslant 0 \quad \text { where } h(s)=A(s)\left\{\int_{s}^{1} A(\tau)^{-1} \mathrm{~d} \tau\right\}^{2} .
$$

Then the operator $T: H_{A} \rightarrow H_{A}$ has no eigenvalues and $u \equiv 0$ is the only solution of (1.1)-(1.3).

REMARK 5.11. We note that $h \in C^{1}((0,1])$, with $h^{\prime} \leqslant 0$ on $(0,1], h(1)=0$ and $\lim _{s \rightarrow 0} h(s)=1 / L$. Hence there is an interval $(a, b)$ with $a<b$ such that $h^{\prime}<0$ on $(a, b)$.

REMARK 5.12. For the profile $L s^{2}$, we find that $h(s)=(1-s)^{2} / L$, and so (5.9) is satisfied, confirming the conclusion of corollary 5.2 (iii). The property (5.9) can also be checked for profiles like $L s^{2}+C s^{q}$, where $C>0$ and $q>2$.

REMARK 5.13. Let us reformulate condition (5.9) in terms of the physical variables (1.10) and (1.11) for the problem of a column buckling under its own weight. Suppose that (1.16) holds with $r=q+3$, so that $p=2$. Then $A \in C^{1}([0,1])$, with $\lim _{t \rightarrow 0} A^{\prime}(t) / t=2 L$, provided that $I \in C^{1}([0,1])$ and that $\lim _{s \rightarrow 0} I^{\prime}(1-s) / s^{r-1}$ exists. We find that $\mathrm{d} h / \mathrm{d} t \leqslant 0$ for all $t \in(0,1)$ if and only if

$$
\frac{\mathrm{d}}{\mathrm{d} z}\left\{I(z)\left[\int_{0}^{z} I(\tau)^{-1} \mathrm{~d} \tau\right]^{2} \int_{z}^{1} S(\tau) \mathrm{d} \tau\right\} \geqslant 0 \quad \text { for all } z \in(0,1) .
$$

Proof. Let $u$ be a solution of (1.1)-(1.3). We introduce new variables by setting

$$
t=\int_{s}^{1} A(\tau)^{-1} \mathrm{~d} \tau \text { and } \quad v(t)=u(s) .
$$


Then $t$ increases from 0 to $\infty$ as $s$ decreases from 1 to 0 . Furthermore, $v \in C^{2}((0,1])$, with $v^{\prime}(t)=-A(s) u^{\prime}(s)$,

$$
\left.\begin{array}{c}
v^{\prime \prime}(t)+\mu \rho(t) v(t)=0 \quad \text { for } 0<t<\infty, \\
v(0)=\lim _{t \rightarrow \infty} v^{\prime}(t)=0, \\
\int_{0}^{1} A(s) u^{\prime}(s)^{2} \mathrm{~d} s=\int_{0}^{\infty} v^{\prime}(t)^{2} \mathrm{~d} t<\infty
\end{array}\right\}
$$

where $\rho(t)=A(s)$.

By lemma 3.3, we know that $u=\mu T u$ and hence $\langle u, u\rangle_{A}=\mu\langle T u, u\rangle_{A}$, which becomes

$$
\int_{0}^{\infty} v^{\prime}(t)^{2} \mathrm{~d} t=\mu \int_{0}^{1} u(s)^{2} \mathrm{~d} s=\mu \int_{0}^{\infty} \rho(t) v(t)^{2} \mathrm{~d} t .
$$

Multiplying (5.10) by $t v^{\prime}(t)$ and integrating from 0 to $T<\infty$, we have that

$$
T v^{\prime}(T)^{2}+\mu T \rho(T) v(T)^{2}=\int_{0}^{T} v^{\prime}(t)^{2}+\mu[t \rho(t)]^{\prime} v(t)^{2} \mathrm{~d} t,
$$

where

$$
\frac{\mathrm{d}}{\mathrm{d} t}[t \rho(t)]=A(s)-t(s) A(s) \frac{\mathrm{d}}{\mathrm{d} s} A(s),
$$

and so, if $T=t(S)$,

$$
\begin{aligned}
\int_{0}^{T}\left|[t \rho(t)]^{\prime}\right| v(t)^{2} \mathrm{~d} t & \leqslant \int_{S}^{1}\left\{A(s)+t(s) A(s)\left|A^{\prime}(s)\right|\right\} u(s)^{2} A(s)^{-1} \mathrm{~d} s \\
& =\int_{S}^{1}\left\{1+t(s)\left|A^{\prime}(s)\right|\right\} u(s)^{2} \mathrm{~d} s .
\end{aligned}
$$

But $\lim _{s \rightarrow 0} t(s)\left|A^{\prime}(s)\right|=2$, since

$$
\lim _{s \rightarrow 0} s \int_{s}^{1} A(\tau)^{-1} \mathrm{~d} \tau=\frac{1}{L} .
$$

Hence there is a constant $C$ such that $t(s)\left|A^{\prime}(s)\right| \leqslant C$ for all $s \in(0,1]$. Using this estimate and lemma 2.5, we see from (5.13) that

$$
\int_{0}^{\infty}\left|[t \rho(t)]^{\prime}\right| v(t)^{2} \mathrm{~d} t<\infty
$$

and consequently $v^{\prime}(t)^{2}+\mu[t \rho(t)]^{\prime} v(t)^{2}$ is integrable on $(0, \infty)$. Let

$$
M \equiv \int_{0}^{\infty} v^{\prime}(t)^{2}+\mu[t \rho(t)]^{\prime} v(t)^{2} \mathrm{~d} t .
$$

It follows from (5.12) that

$$
\lim _{T \rightarrow \infty}\left\{T v^{\prime}(T)^{2}+\mu T \rho(T) v(T)^{2}\right\}=M<\infty .
$$

But

$$
0 \leqslant \int_{0}^{T} \rho(t) v(t)^{2} \mathrm{~d} t=\int_{S}^{1} u(s)^{2} \mathrm{~d} s \leqslant 4\|u\|_{2}^{2}
$$


by lemma 2.5. This implies that $\left[v^{\prime}\right]^{2}+\mu \rho v^{2}$ is integrable on $(0, \infty)$, and hence that $M=0$. Recalling (5.11), we now have that

$$
\mu \int_{0}^{\infty} \rho(t) v(t)^{2} \mathrm{~d} t=\int_{0}^{\infty} v^{\prime}(t)^{2} \mathrm{~d} t=-\int_{0}^{\infty} \mu[t \rho(t)]^{\prime} v(t)^{2} \mathrm{~d} t
$$

which yields

$$
\int_{0}^{\infty} t^{-1}\left[t^{2} \rho(t)\right]^{\prime} v(t)^{2} \mathrm{~d} t=0
$$

But

$$
\frac{\mathrm{d}}{\mathrm{d} t}\left[t^{2} \rho(t)\right]=-A(s) \frac{\mathrm{d}}{\mathrm{d} s} h(s) \geqslant 0
$$

by $(5.9)$, and so $\left[t^{2} \rho(t)\right]^{\prime} v(t)^{2}=0$ on $(0, \infty)$. Hence $v(t)=0$ on the interval $(t(b), t(a))$, where $(a, b)$ is the interval discussed in remark 5.11. Since $v$ satisfies the differential equation (5.10), this implies that $v \equiv 0$ on $(0, \infty)$ and the proof is complete.

\section{The differential operator}

In $\S 3$ we defined the operator $T: H_{A} \rightarrow H_{A}$ associated with the boundary-value problem (1.1)-(1.3). As we show in [16], the space $H_{A}$ is the natural setting for the study of the nonlinear eigenvalue problems posed by (1.5) or (1.14) with (1.2) and (1.3). The operator $T$ plays a important role in the discussion of these problems, since $I-\mu T: H_{A} \rightarrow H_{A}$ constitutes the linear approximation. However, confronted directly with the linear eigenvalue problem (1.1) and (1.2), there is another standard way of formulating a rigorous spectral theory for such a boundary-value problem. It involves using (1.1) and (1.2) to define an unbounded self-adjoint operator $N$ in the usual Hilbert space $L^{2}(0,1)$. In this section we explore this possibility and show that it leads to exactly the same spectrum and essential spectrum as the approach based on $T$. Indeed, we establish a precise relation of similarity between the operators $T$ and $N$ and we show that $H_{A}$ is characterized as the graph space of the positive square root $N^{1 / 2}$ of $N$.

For the rest of this section let $A$ be a profile with tapering of order $p$, where $0 \leqslant p \leqslant 2$. We begin by considering the set

$$
D=\left\{u \in L^{2}(0,1):\left(A u^{\prime}\right)^{\prime} \in L^{2}(0,1)\right\}
$$

formed by the elements $u \in L^{2}(0,1)$ that have a generalized derivative $u^{\prime}$, which has the property that $A u^{\prime}$ has a generalized derivative belonging to $L^{2}(0,1)$. We claim that, after modification on a set of measure zero,

$$
u \in C^{1}((0,1]) \text { and } A u^{\prime} \in C([0,1]) .
$$

In fact, setting

$$
f=\left(A u^{\prime}\right)^{\prime} \text { and } g=A u^{\prime} \text { for some } u \in D,
$$

we have that $g \in L_{\text {loc }}^{1}(0,1)$, with $g^{\prime}=f \in L^{2}(0,1) \subset L^{1}(0,1)$. It follows from this that, after modification on a set of measure zero,

$$
A u^{\prime}=g \in C([0,1]) \text { and } g(s)=g(t)+\int_{t}^{s} f(\tau) \mathrm{d} \tau
$$


for all $s, t \in[0,1]$. In particular,

$$
A(s) u^{\prime}(s)=\alpha+\int_{0}^{s} f(\tau) \mathrm{d} \tau \quad \text { for all } s \in[0,1],
$$

where $\alpha=g(0)=\left[A u^{\prime}\right](0)$. Thus, for $0<s \leqslant 1$,

$$
u^{\prime}(s)=\frac{1}{A(s)}\left\{\alpha+\int_{0}^{s} f(\tau) \mathrm{d} \tau\right\}
$$

showing that $u \in C^{1}((0,1])$.

By (6.2) we can define a subset of $D$ by setting

$$
D_{0}=\left\{u \in D:\left[A u^{\prime}\right](0)=0 \text { and } u(1)=0\right\} .
$$

LEMMA 6.1.

(i) $D_{0} \subset H_{A}$ and

$$
\|u\|_{A} \leqslant \frac{2}{\sqrt{K_{2}}}\left|\left(A u^{\prime}\right)^{\prime}\right|_{2} \quad \text { for all } u \in D_{0},
$$

where $K_{2} \equiv \inf \left\{A(s) / s^{p}: 0 \leqslant s \leqslant 1\right\}>0$.

(ii) $D_{0}$ is dense in the Hilbert space $\left(H_{A},\langle\cdot, \cdot\rangle_{A}\right)$.

(iii) For all $u \in D_{0}$ and $v \in H_{A}$,

$$
\lim _{s \rightarrow 0} A(s) u^{\prime}(s) v(s)=0 \quad \text { and } \quad \int_{0}^{1}\left(A u^{\prime}\right)^{\prime} v \mathrm{~d} s=-\int_{0}^{1} A u^{\prime} v^{\prime} \mathrm{d} s .
$$

Proof. For any $u \in D_{0}$, it follows from (6.3) that

$$
\begin{aligned}
A(s) u^{\prime}(s)^{2} & =\frac{1}{A(s)}\left\{\int_{0}^{s} f(\tau) \mathrm{d} \tau\right\}^{2} \\
& \leqslant \frac{1}{K_{2} s^{p}}\left\{\int_{0}^{s} f(\tau) \mathrm{d} \tau\right\}^{2} \\
& \leqslant \frac{1}{K_{2} s^{2}}\left\{\int_{0}^{s} f(\tau) \mathrm{d} \tau\right\}^{2}
\end{aligned}
$$

for all $s \in(0,1]$, since $p \leqslant 2$. But, arguing as for Hardy's inequality,

$$
\begin{aligned}
\int_{\varepsilon}^{1} s^{-2}\left\{\int_{0}^{s} f(\tau) \mathrm{d} \tau\right\}^{2} \mathrm{~d} s \\
\leqslant \varepsilon^{-1}\left\{\int_{0}^{\varepsilon} f(\tau) \mathrm{d} \tau\right\}^{2}+\int_{\varepsilon}^{1} s^{-1} 2\left\{\int_{0}^{s} f(\tau) \mathrm{d} \tau\right\} f(s) \mathrm{d} s \\
\leqslant \int_{0}^{\varepsilon} f(\tau)^{2} \mathrm{~d} \tau+2\left[\int_{\varepsilon}^{1} s^{-2}\left\{\int_{0}^{s} f(\tau) \mathrm{d} \tau\right\}^{2} \mathrm{~d} s\right]^{1 / 2}\left[\int_{\varepsilon}^{1} f(\tau)^{2} \mathrm{~d} \tau\right]^{1 / 2}
\end{aligned}
$$

for all $\varepsilon \in(0,1)$. Since $f \in L^{2}(0,1)$,

$$
\int_{0}^{\varepsilon} f(\tau)^{2} \mathrm{~d} \tau \rightarrow 0 \quad \text { as } \varepsilon \rightarrow 0
$$


and it follows easily that

$$
\int_{0}^{1} s^{-2}\left\{\int_{0}^{s} f(\tau) \mathrm{d} \tau\right\}^{2} \mathrm{~d} s \leqslant 4 \int_{0}^{1} f(\tau)^{2} \mathrm{~d} \tau .
$$

Returning to (6.5), we see that part (i) follows immediately.

(ii) Let $u \in H_{A}$ and choose any $\delta>0$. Define $v$ by setting

$$
v(s)= \begin{cases}u(\delta) & \text { for } 0 \leqslant s \leqslant \delta \\ u(s) & \text { for } \delta<s \leqslant 1\end{cases}
$$

It follows that $v \in H_{A}$, with

$$
\|u-v\|_{A}^{2}=\int_{0}^{\delta} A(s) u^{\prime}(s)^{2} \mathrm{~d} s .
$$

Since $A^{1 / 2} v^{\prime} \in L^{2}(0,1)$ and $A \in C([0,1])$, we have that $A v^{\prime} \in L^{2}(\delta, 1)$. Hence there exists a sequence $\left\{w_{k}\right\} \subset C_{0}^{\infty}((\delta, 1))$ such that

$$
\int_{\delta}^{1}\left(w_{k}-A v^{\prime}\right)^{2} \mathrm{~d} s \rightarrow 0 \quad \text { as } k \rightarrow \infty .
$$

Setting $w_{k}(s)=0$ for $s \in[0, \delta]$, we have that $w_{k} \in C_{0}^{\infty}((0,1))$ and $w_{k} / A \in C([0,1])$. Thus we can define a function $v_{k}$ on $[0,1]$ by

$$
v_{k}(s)=-\int_{s}^{1} \frac{w_{k}(t)}{A(t)} \mathrm{d} t
$$

Clearly, $v_{k} \in C^{1}([0,1])$, with $A v_{k}^{\prime}=w_{k} \in C_{0}^{\infty}((0,1))$. Furthermore, $A(0) v_{k}^{\prime}(0)=0$ and $v_{k}(1)=0$. Thus $v_{k} \in D_{0}$ and

$$
\begin{aligned}
\left\|v_{k}-v\right\|_{A}^{2} & =\int_{0}^{1} A\left[v_{k}^{\prime}-v^{\prime}\right]^{2} \mathrm{~d} s \\
& =\int_{\delta}^{1} A\left[v_{k}^{\prime}-v^{\prime}\right]^{2} \mathrm{~d} s \\
& =\int_{\delta}^{1} \frac{1}{A}\left[A v_{k}^{\prime}-A v^{\prime}\right]^{2} \mathrm{~d} s \\
& =\int_{\delta}^{1} \frac{1}{A}\left[w_{k}-A v^{\prime}\right]^{2} \mathrm{~d} s \\
& \leqslant \frac{1}{K_{2} \delta^{p}} \int_{\delta}^{1}\left[w_{k}-A v^{\prime}\right]^{2} \mathrm{~d} s
\end{aligned}
$$

Thus, combining this with (6.7), we have that

$$
\left\|v_{k}-u\right\|_{A} \leqslant\left\{\int_{0}^{\delta} A(s) u^{\prime}(s)^{2} \mathrm{~d} s\right\}^{1 / 2}+\left\{\frac{1}{K_{2} \delta^{p}} \int_{\delta}^{1}\left[w_{k}-A v^{\prime}\right]^{2} \mathrm{~d} s\right\}^{1 / 2} .
$$

Now, given $u \in H_{A}$ and $\varepsilon>0$, we can choose $\delta>0$ such that

$$
\left\{\int_{0}^{\delta} A(s) u^{\prime}(s)^{2} \mathrm{~d} s\right\}^{1 / 2}<\varepsilon
$$


and, for this choice of $\delta$, it follows from (6.8) that there exists $k \in \mathbb{N}$ such that

$$
\left\{\frac{1}{K_{2} \delta^{p}} \int_{\delta}^{1}\left[w_{k}-A v^{\prime}\right]^{2} \mathrm{~d} s\right\}^{1 / 2}<\varepsilon .
$$

Thus $\left\|v_{k}-u\right\|_{A}<2 \varepsilon$, where $v_{k} \in D_{0}$, showing that $D_{0}$ is dense in $H_{A}$.

(iii) Let $u, v \in D_{0}$ and set $f=\left(A u^{\prime}\right)^{\prime}$ and $g=\left(A v^{\prime}\right)^{\prime}$. Then $f, g \in L^{2}(0,1)$ and we have that

$$
\begin{aligned}
\int_{0}^{1} f(s) v(s) \mathrm{d} s & =\lim _{\varepsilon \rightarrow 0} \int_{\varepsilon}^{1} f(s) v(s) \mathrm{d} s \\
& =\lim _{\varepsilon \rightarrow 0}\left\{-A(\varepsilon) u^{\prime}(\varepsilon) v(\varepsilon)-\int_{\varepsilon}^{1} A(s) u^{\prime}(s) v^{\prime}(s) \mathrm{d} s\right\},
\end{aligned}
$$

so, since $u, v \in H_{A}$, we see that $\lim _{\varepsilon \rightarrow 0} A(\varepsilon) u^{\prime}(\varepsilon) v(\varepsilon)=l$, where

$$
l=-\left\{\langle u, v\rangle_{A}+\langle f, v\rangle\right\} \in \mathbb{R} .
$$

Now

$$
A(s) u^{\prime}(s)=\int_{0}^{s} f(t) \mathrm{d} t
$$

and so

$$
\left|A(s) u^{\prime}(s)\right| \leqslant s^{1 / 2}\left\{\int_{0}^{s} f(t)^{2} \mathrm{~d} t\right\}^{1 / 2} \leqslant s^{1 / 2}\left\{\int_{0}^{1} f(t)^{2} \mathrm{~d} t\right\}^{1 / 2} .
$$

Using the estimate (2.1) or (2.2), it follows that $l=0$ if $p<2$. For the case $p=2$, we observe that if $l \neq 0$, then $s^{-1} A(s) u^{\prime}(s) v(s) \notin L^{1}(0,1)$. But

$$
\begin{aligned}
\int_{0}^{1}\left|s^{-1} A(s) u^{\prime}(s) v(s)\right| \mathrm{d} s & \leqslant\left\{\int_{0}^{1} A(s) u^{\prime}(s)^{2} \mathrm{~d} s\right\}^{1 / 2}\left\{\int_{0}^{1} s^{-2} A(s) v(s)^{2} \mathrm{~d} s\right\}^{1 / 2} \\
& \leqslant\|u\|_{A} \sqrt{K_{1}}|v|_{2}<\infty
\end{aligned}
$$

so $l=0$. Thus $l=0$ for all $p \in[0,2]$ and hence

$$
\int_{0}^{1} A(s) u^{\prime}(s) v^{\prime}(s) \mathrm{d} s=-\int_{0}^{1} f(s) v(s) \mathrm{d} s .
$$

We now introduce the differential operator in $N: D(N) \subset L^{2}(0,1) \rightarrow L^{2}(0,1)$ associated with problem (1.1) and (1.2) by setting

$$
D(N)=D_{0} \quad \text { and } \quad N(u)=-\left(A u^{\prime}\right)^{\prime} \quad \text { for } u \in D(N) .
$$

Let $\langle\cdot, \cdot\rangle$ denote the usual scalar product on $L^{2}(0,1)$.

TheOREM 6.2. $D_{0}$ is a dense subspace of $\left(L^{2}(0,1),\langle\cdot, \cdot\rangle\right)$ and

$$
N: D(N) \subset L^{2}(0,1) \rightarrow L^{2}(0,1)
$$

is a positive self-adjoint operator. Furthermore,

$$
\langle N(u), v\rangle=\int_{0}^{1} A(s) u^{\prime}(s) v^{\prime}(s) \mathrm{d} s=\langle u, v\rangle_{A} \quad \text { for all } u \in D(N) \text { and } v \in H_{A},
$$


and $\Lambda(A)=\inf \sigma(N)$, where $\sigma(N)$ denotes the spectrum of $N$.

Proof. Let $w \in C_{0}^{\infty}((0,1))$. Then define a function $V(w)$ by setting

$$
V(w)(s)=-\int_{s}^{1} \frac{w(t)}{A(t)} \mathrm{d} t \quad \text { for } 0 \leqslant s \leqslant 1 .
$$

It follows that $V(w) \in C^{1}([0,1])$, with $V(w)^{\prime}(s)=w(s) / A(s)$. Thus $V(w)(1)=0$, $A(0) V(w)^{\prime}(0)=w(0)=0$ and $\left[A V(w)^{\prime}\right]^{\prime}=w^{\prime} \in C_{0}^{\infty}((0,1))$, showing that $V(w) \in D_{0}$ for all $w \in C_{0}^{\infty}((0,1))$.

Suppose now that $v \in L^{2}(0,1)$ and that $\langle v, u\rangle=0$ for all $u \in D_{0}$. Then $\langle v, V(w)\rangle=0$ for all $w \in C_{0}^{\infty}((0,1))$. Thus, for all $w \in C_{0}^{\infty}((0,1))$,

$$
\begin{aligned}
0 & =\int_{0}^{1} v(s) \int_{s}^{1} \frac{w(t)}{A(t)} \mathrm{d} t \mathrm{~d} s \\
& =\left.\int_{0}^{s} v(\tau) \mathrm{d} \tau \int_{s}^{1} \frac{w(t)}{A(t)} \mathrm{d} t\right|_{s=0} ^{s=1}+\int_{0}^{1} \int_{0}^{s} v(\tau) \mathrm{d} \tau \frac{w(s)}{A(s)} \mathrm{d} s \\
& =\int_{0}^{1} \int_{0}^{s} v(\tau) \mathrm{d} \tau \frac{w(s)}{A(s)} \mathrm{d} s
\end{aligned}
$$

which implies that

$$
\int_{0}^{s} v(\tau) \mathrm{d} \tau=0 \quad \text { on }(0,1), \text { and consequently } v(s)=0 \text { on }(0,1) .
$$

This proves that $D_{0}$ is dense in $L^{2}(0,1)$.

The adjoint operator $N^{*}: D\left(N^{*}\right) \subset L^{2}(0,1) \rightarrow L^{2}(0,1)$ is now defined by

$$
\begin{aligned}
& D\left(N^{*}\right)=\left\{v \in L^{2}(0,1): \exists z \in L^{2}(0,1)\right. \text { such that } \\
& \qquad\langle N(u), v\rangle=\langle u, z\rangle \text { for all } u \in D(N)\}
\end{aligned}
$$

and

$$
N^{*}(v)=z \quad \text { for all } v \in D\left(N^{*}\right) .
$$

Let us show that $D\left(N^{*}\right)=D_{0}$. In fact, by lemma 6.1(iii), we know that for all $u, v \in D_{0}$,

$$
\langle N(u), v\rangle=\langle u, v\rangle_{A}=\langle u, N(v)\rangle,
$$

proving that $D_{0} \subset D\left(N^{*}\right)$ and that $N^{*}(v)=N(v)$ for all $v \in D(N)$. Thus we need only show that $D\left(N^{*}\right) \subset D_{0}$. To this end, suppose that $v \in D\left(N^{*}\right)$ and that $z \in L^{2}(0,1)$ is such that $\langle N(u), v\rangle=\langle u, z\rangle$ for all $u \in D(N)$. Then

$$
-\int_{0}^{1}\left(A u^{\prime}\right)^{\prime} v \mathrm{~d} s=\int_{0}^{1} u z \mathrm{~d} s \text { for all } u \in D(N) .
$$

In particular, for any $w \in C_{0}^{\infty}((0,1))$,

$$
-\int_{0}^{1}\left(A[V(w)]^{\prime}\right)^{\prime} v \mathrm{~d} s=\int_{0}^{1} V(w) z \mathrm{~d} s
$$


where $V(w)$ is defined by (6.10) and so

$$
-\int_{0}^{1} w^{\prime} v \mathrm{~d} s=-\int_{0}^{1}\left\{\int_{s}^{1} \frac{w(t)}{A(t)} \mathrm{d} t\right\} z(s) \mathrm{d} s=-\int_{0}^{1} \frac{w(s)}{A(s)} \int_{0}^{s} z(t) \mathrm{d} t \mathrm{~d} s .
$$

Thus $v$ has a generalized derivative on $(0,1)$ and

$$
v^{\prime}(s)=-\frac{1}{A(s)} \int_{0}^{s} z(t) \mathrm{d} t
$$

It follows that $A v^{\prime} \in C([0,1])$ and $A(0) v^{\prime}(0)=0$. Since $\left(A v^{\prime}\right)^{\prime}=-z \in L^{2}(0,1)$, we need only show that $v(1)=0$ in order to establish that $v \in D_{0}$. For this, let $\hat{v}=v-v(1)$. Clearly, $\hat{v} \in D_{0}$. For all $u \in D_{0}$, we have that

$$
\begin{aligned}
\int_{0}^{1} u z \mathrm{~d} s & =-\int_{0}^{1}\left(A u^{\prime}\right)^{\prime} v \mathrm{~d} s \\
& =-\int_{0}^{1}\left(A u^{\prime}\right)^{\prime}[\hat{v}+v(1)] \mathrm{d} s \\
& =-\int_{0}^{1}\left(A u^{\prime}\right)^{\prime} \hat{v} \mathrm{~d} s-v(1) \int_{0}^{1}\left(A u^{\prime}\right)^{\prime} \mathrm{d} s \\
& =\langle N(u), \hat{v}\rangle-v(1) A(1) u^{\prime}(1),
\end{aligned}
$$

But, by lemma 6.1(iii),

$$
\langle N(u), \hat{v}\rangle=\langle u, N(\hat{v})\rangle=-\int_{0}^{1} u\left(A v^{\prime}\right)^{\prime} \mathrm{d} s=\langle u, z\rangle,
$$

since $u, \hat{v} \in D_{0}$ and $\left(A \hat{v}^{\prime}\right)^{\prime}=\left(A v^{\prime}\right)^{\prime}=-z$. Therefore, $v(1) A(1) u^{\prime}(1)=0$ for all $u \in D_{0}$ and it follows that $v(1)=0$. This proves that $v \in D_{0}$ and hence that $D\left(N^{*}\right) \subset D_{0}$. Thus $D\left(N^{*}\right)=D_{0}$ and the self-adjointness of $N$ is established.

By lemma 6.1(iii), $\langle N(u), v\rangle=\langle u, v\rangle_{A}$ for all $u \in D(N)$ and $v \in H_{A}$. In particular, for all $u \in D(N)$,

$$
\langle N(u), u\rangle=\langle u, u\rangle_{A} \geqslant \Lambda(A)\langle u, u\rangle,
$$

where $\Lambda(A)>0$, since $p \leqslant 2$ by (3.1). This proves that $N \geqslant \Lambda(A) I$, so $N$ is positive and, by theorem 4.4 in ch. III of [5],

$$
\inf \sigma(N)=\inf \left\{\langle N(u), u\rangle: u \in D(N) \text { and }|u|_{2}=1\right\} .
$$

Since $D(N) \subset H_{A}$ and $\langle N(u), u\rangle=\langle u, u\rangle_{A}$ for all $u \in D(N)$,

$$
\begin{aligned}
& \inf \left\{\langle N(u), u\rangle: u \in D(N) \text { and }|u|_{2}=1\right\} \\
& \qquad \inf \left\{\langle u, u\rangle_{A}: u \in H_{A} \text { and }|u|_{2}=1\right\}=\Lambda(A) .
\end{aligned}
$$

But, for any $u \in H_{A}$ with $|u|_{2}=1$, there is a sequence $\left\{u_{n}\right\} \subset D(N)$ such that $\left\|u-u_{n}\right\|_{A} \rightarrow 0$, since $D(N)$ is dense in $H_{A}$ by lemma $6.1(\mathrm{ii})$. Then

$$
\left\langle N\left(u_{n}\right), u_{n}\right\rangle=\left\|u_{n}\right\|_{A}^{2} \rightarrow\|u\|_{A}^{2} \quad \text { and } \quad\left|u_{n}\right|_{2} \rightarrow|u|_{2}
$$


by lemma 2.5 , since $p \leqslant 2$. Hence $v_{n}=u_{n} /\left|u_{n}\right|_{2} \in D(N)$, with $\left|v_{n}\right|_{2}=1$ and

$$
\left\langle N\left(v_{n}\right), v_{n}\right\rangle=\frac{\left\langle N\left(u_{n}\right), u_{n}\right\rangle}{\left|u_{n}\right|_{2}^{2}} \rightarrow \frac{\|u\|_{A}^{2}}{|u|_{2}^{2}}=\|u\|_{A}^{2},
$$

showing that

$$
\inf \left\{\langle N(u), u\rangle: u \in D(N) \text { and }|u|_{2}=1\right\}=\inf \left\{\langle u, u\rangle_{A}: u \in H_{A} \text { and }|u|_{2}=1\right\} .
$$

Thus

$$
\inf \sigma(N)=\inf \left\{\langle u, u\rangle_{A}: u \in H_{A} \text { and }|u|_{2}=1\right\}=\Lambda(A) .
$$

This completes the proof of the theorem.

Since $N$ is a positive self-adjoint operator, it has a unique positive self-adjoint square root, which we denote by $N^{1 / 2}: D\left(N^{1 / 2}\right) \subset L^{2}(0,1) \rightarrow L^{2}(0,1)$. Then $\left(D\left(N^{1 / 2}\right),\langle\cdot, \cdot\rangle_{N^{1 / 2}}\right)$ is a Hilbert space, called the graph space of $N^{1 / 2}$ or the form space of $N$, where

$$
\langle u, v\rangle_{N^{1 / 2}}=\langle u, v\rangle+\left\langle N^{1 / 2}(u), N^{1 / 2}(v)\right\rangle \quad \text { for all } u, v \in D\left(N^{1 / 2}\right) .
$$

The set $D(N)$ is a dense subspace of $\left(D\left(N^{1 / 2}\right),\langle\cdot, \cdot\rangle_{N^{1 / 2}}\right)$.

We now recall one of the main results in [14] concerning arbitrary self-adjoint operators, in the simpler context of the positive bounded self-adjoint operator $N$.

\section{Proposition 6.3.}

(i) There is a unique bounded linear operator $B$ from $\left(D\left(N^{1 / 2}\right),\langle\cdot, \cdot\rangle_{N^{1 / 2}}\right)$ into itself such that

$$
\langle N(u), v\rangle=\langle B(u), v\rangle_{N^{1 / 2}} \quad \text { for all } u \in D(N) \text { and } v \in D\left(N^{1 / 2}\right) .
$$

(ii) There is a unique bounded linear operator $L$ from $\left(D\left(N^{1 / 2}\right),\langle\cdot, \cdot\rangle_{N^{1 / 2}}\right)$ into itself such that

$$
\langle u, v\rangle=\langle L(u), v\rangle_{N^{1 / 2}} \quad \text { for all } u, v \in D\left(N^{1 / 2}\right) .
$$

(iii) $\sigma(N)=\left\{\lambda \in \mathbb{R}: B-\lambda L: D\left(N^{1 / 2}\right) \rightarrow D\left(N^{1 / 2}\right)\right.$ is not an isomorphism $\}$.

(iv) $\sigma_{\mathrm{e}}(N)=\left\{\lambda \in \mathbb{R}: B-\lambda L: D\left(N^{1 / 2}\right) \rightarrow D\left(N^{1 / 2}\right)\right.$ is not a Fredholm operator $\}$.

Proof. See theorem 3.3 of [14].

We can now relate the spectrum of $N$ to that of the bounded self-adjoint operator $T$ in $\left(H_{A},\langle\cdot, \cdot\rangle_{A}\right)$ defined in proposition 3.1. First of all, we show that the Hilbert spaces $\left(H_{A},\langle\cdot, \cdot\rangle_{A}\right)$ and $\left(D\left(N^{1 / 2}\right),\langle\cdot, \cdot\rangle_{N^{1 / 2}}\right)$ are the same, up to equivalence of norms. In fact, for $u \in D(N)$,

$$
\begin{aligned}
\|u\|_{N^{1 / 2}}^{2} & =\langle u, u\rangle_{N^{1 / 2}} \\
& =\langle u, u\rangle+\left\langle N^{1 / 2}(u), N^{1 / 2}(u)\right\rangle \\
& =|u|_{2}^{2}+\langle N(u), u\rangle \\
& =|u|_{2}^{2}+\|u\|_{A}^{2},
\end{aligned}
$$


so that

$$
\|u\|_{A}^{2} \leqslant\|u\|_{N^{1 / 2}}^{2} \leqslant\left\{1+\frac{1}{\Lambda(A)}\right\}\|u\|_{A}^{2} .
$$

This means that $\|\cdot\|_{A}$ and $\|\cdot\|_{N^{1 / 2}}$ are equivalent norms on $D(N)$. But $D(N)$ is a dense subspace of both $\left(H_{A},\langle\cdot, \cdot\rangle_{A}\right)$ and $\left(D\left(N^{1 / 2}\right),\langle\cdot, \cdot\rangle_{N^{1 / 2}}\right)$, so it follows easily that

$$
H_{A}=D\left(N^{1 / 2}\right), \quad \text { with }\langle u, v\rangle_{N^{1 / 2}}=\langle u, v\rangle+\langle u, v\rangle_{A}
$$

for all $u, v \in H_{A}$. In particular, $\|\cdot\|_{A}$ and $\|\cdot\|_{N^{1 / 2}}$ are equivalent norms on $H_{A}$.

Notice that (6.13) can be written as

$$
\langle u, v\rangle_{N^{1 / 2}}=\langle T(u), v\rangle_{A}+\langle u, v\rangle_{A}=\langle(T+I) u, v\rangle_{A}
$$

for all $u, v \in H_{A}$, by the definition of $T$.

TheOREM 6.4. For all $\lambda \in \mathbb{R} \backslash\{0\}$, we have the following.

(i) $N-\lambda I: D(N) \rightarrow L^{2}(0,1)$ is an isomorphism $\Longleftrightarrow T-1 / \lambda I: H_{A} \rightarrow H_{A}$ is an isomorphism.

(ii) $N-\lambda I: D(N) \rightarrow L^{2}(0,1)$ is a Fredholm operator $\Longleftrightarrow T-1 / \lambda I: H_{A} \rightarrow H_{A}$ is a Fredholm operator.

Furthermore, for $0 \notin \sigma(N)$,

$$
\sigma(T)=\left\{\mu \in \mathbb{R} \backslash\{0\}: \frac{1}{\mu} \in \sigma(N)\right\} \cup\{0\}
$$

and

$$
\sigma_{\mathrm{e}}(T)=\left\{\mu \in \mathbb{R} \backslash\{0\}: \frac{1}{\mu} \in \sigma_{\mathrm{e}}(N)\right\} \cup\{0\} .
$$

Proof. We begin by relating $T$ to the operators $B$ and $L$ defined in proposition 6.3. Since $D(N)$ is a dense subspace of $\left(D\left(N^{1 / 2}\right),\langle\cdot, \cdot\rangle_{N^{1 / 2}}\right)$ and $\langle B(u), v\rangle_{N^{1 / 2}}=$ $\langle N(u), v\rangle=\langle u, v\rangle_{A}$ for all $u \in D(N)$ and $v \in D\left(N^{1 / 2}\right)$, it follows that

$$
\langle B(u), v\rangle_{N^{1 / 2}}=\langle u, v\rangle_{A} \quad \text { for all } u, v \in H_{A} .
$$

Using (6.14), we now have that

$$
\langle u, v\rangle_{A}=\langle B(u), v\rangle_{N^{1 / 2}}=\langle(T+I) B(u), v\rangle_{A}
$$

and

$$
\langle T(u), v\rangle_{A}=\langle u, v\rangle=\langle L(u), v\rangle_{N^{1 / 2}}=\langle(T+I) L(u), v\rangle_{A}
$$

for all $u, v \in H_{A}$. Hence

$$
I=(T+I) B \quad \text { and } \quad T=(T+I) L .
$$

This means that for any $\lambda \in \mathbb{R}, I-\lambda T=(T+I)(B-\lambda L)$. But we know from proposition 3.1 that $T$ is a bounded positive self-adjoint operator on the Hilbert space $\left(H_{A},\langle\cdot, \cdot\rangle_{A}\right)$, so $T+I: H_{A} \rightarrow H_{A}$ is an isomorphism. Thus $I-\lambda T: H_{A} \rightarrow H_{A}$ 
is an isomorphism if and only if $(B-\lambda L): H_{A} \rightarrow H_{A}$ is an isomorphism, and $I-\lambda T: H_{A} \rightarrow H_{A}$ is a Fredholm operator if and only if $(B-\lambda L): H_{A} \rightarrow H_{A}$ is a Fredholm operator. The conclusions (i) and (ii) now follow from proposition 6.3. By theorem $6.2,0 \notin \sigma(N)$ and the proof is complete.

Remark 6.5. Since $0 \notin \sigma(N), \operatorname{rge}(N)=L^{2}(0,1)$ and $N^{-1}: L^{2}(0,1) \rightarrow D(N)$ is well defined. By theorem 6.2,

$$
\left\langle N^{-1}(z), v\right\rangle_{A}=\left\langle N N^{-1}(z), v\right\rangle \quad \text { for all } z \in L^{2}(0,1) \text { and } v \in H_{A} .
$$

Thus

$$
\left\langle N^{-1}(z), v\right\rangle_{A}=\langle z, v\rangle=\langle T z, v\rangle_{A}
$$

for all $z, v \in H_{A}$. Hence $T=\left.N^{-1}\right|_{H_{A}}$.

\section{Acknowledgments}

I am grateful to Professor S. J. Cox for drawing my attention to the absence of results about bifurcation for a tapered Euler column.

\section{References}

1 S. S. Antman. Nonlinear problems of elasticity (Springer, 1995).

2 D. Bernoulli. Methodus universalis determinandae curvaturae fili a potentiis quamcunque legem inter se observantibus extensi, una cum solutione problematum quorundam novorum eo pertinentium. Commun. Acad. Sci. Petrop. 8 (1728), 62-69.

3 H. Brézis. Analyse fonctionnelle (Paris: Masson, 1983).

4 S. J. Cox and McCarthy. The shape of the tallest column. SIAM J. Math. Analysis 29 (1998), 547-554.

5 D. E. Edmunds and W. D. Evans. Spectral theory and differential operators (Oxford University Press, 1987).

6 L. Euler. Determinatio onerum, quae columnae gestare valent. Acta Acad. Sci. Petrop. 2 (1778), 121-145.

7 L. Euler. De altitudine columnarum sub proprio pondere corrueuntium. Acta Acad. Sci. Petrop. 2 (1778), 163-193.

8 A. G. Greenhill. Determination of the greatest height consistent with stability that a vertical pole or mast can be made, and of the greatest height to which a tree of given proportions can grow. Proc. Camb. Phil. Soc. 4 (1881), 65-73.

9 G. H. Hardy, J. E. Littlewood and G. Polya. Inequalities (Cambridge University Press, 1934).

10 J. Jost. Postmodern analysis (Springer, 1998).

11 J. B. Keller and F. I. Niordson. The tallest column. J. Math. Mech. 16 (1966), 433-446.

12 A. E. H. Love. A treatise on the mathematical theory of elasticity (New York: Dover, 1944).

13 W. T. Reid. Sturmian theory for ordinary differential equations (Springer, 1980).

14 C. A. Stuart. Spectrum of a self-adjoint operator and Palais-Smale conditions. J. Lond. Math. Soc. 61 (2000), 581-592.

15 C. A. Stuart. Buckling of a tapered elastica. C. R. Acad. Sci. Paris 331 (2000), 417-421.

16 C. A. Stuart. Buckling of a heavy tapered rod. J. Math. Pures Appl. 80 (2001), 281-337.

17 S. P. Timoshenko. Theory of elastic stability, 2nd edn (McGraw-Hill, 1988).

18 C. Truesdell. The rational mechanics of flexible or elastic bodies. In Introduction to Leonhardi Euleri Opera Omnia, Ser. secunda XI, Füssli, Zürich, pp. 1638-1788 (1960).

W. Walter. Ordinary differential equations (Springer, 1998).

(Issued 21 June 2002) 